\title{
MINIMAL SINGULAR METRICS OF A LINE BUNDLE ADMITTING NO ZARISKI DECOMPOSITION
}

\author{
TAKAYUKI KOIKE
}

\begin{abstract}
We give a concrete expression of a minimal singular metric on a big line bundle on a compact Kähler manifold which is the total space of a toric bundle over a complex torus. In this class of manifolds, Nakayama constructed examples which have line bundles admitting no Zariski decomposition even after modifications. As an application, we discuss the Zariski closedness of non-nef loci.
\end{abstract}

\section{INTRODUCTION}

We consider the positivity of a big holomorphic line bundle over a compact Kähler complex manifold. Especially, we are interested in the information related to the obstruction to the nef-ness of the line bundle. Our main result is the explicit construction of a minimal singular metric, or a singular hermitian metric on $L$ with minimal singularities, of a big line bundle $L$ when the manifold $X$ is the total space of a smooth projective toric bundle over a complex torus (Theorem 4.7).

In order to state our main theorem in general form, we have to define some terminology. So in this section, we introduce our result only when $(X, L)$ is a Nakayama example ([14, IV §2.6]), which is one of the most important examples when we study the obstruction to the nef-ness of the line bundle, since it admits no Zariski decomposition even after modifications. Let $E_{1}$ be a sufficiently general smooth elliptic curve such as $\mathbb{C} /(\mathbb{Z}+(\pi+$ $\sqrt{-1}) \mathbb{Z}), E_{2}$ a copy of $E_{1}$, and $z_{j}$ a coordinate of $E_{j}$ for $j=1,2$. Let us fix an integer $a>1$, points $p_{1} \in E_{1}, p_{2} \in E_{2}$, and define the three line bundles $L_{j}(j=0,1,2)$ over $V=E_{1} \times E_{2}$ by

$$
\begin{aligned}
& L_{0}=\mathcal{O}_{V}\left(2 F_{1}-4 F_{2}+2 \Delta\right), \\
& L_{1}=\mathcal{O}_{V}\left((a-1) F_{1}+(a-1) F_{2}+(a+2) \Delta\right), \\
& L_{2}=\mathcal{O}_{V}\left((a+3) F_{1}+(a-3) F_{2}+a \Delta\right),
\end{aligned}
$$

where $F_{1}$ stands for the prime divisor $\left\{p_{1}\right\} \times E_{2} \subset V, F_{2}$ stands for the prime divisor $E_{1} \times\left\{p_{2}\right\} \subset V$, and $\Delta$ stands for the prime divisor $\{(x, y) \in E \times E \mid x=y\}$. Then there exists a hermitian metric $h_{j}$ over $L_{j}$ whose curvature tensor $\Theta_{h_{j}} \in c_{1}\left(L_{j}\right)$ is a harmonic

2010 Mathematics Subject Classification. Primary 32J25; Secondary 32J27, 14C20.

Key words and phrases. Minimal singular metrics, Zariski decompositions, Nakayama example, Kiselman numbers, Lelong numbers, non-nef loci, multiplier ideal sheaves. 
form and each $h_{j}$ can be denoted as $h_{j}(\xi, \eta)_{\left(z_{1}, z_{2}\right)}=e^{-\varphi_{j}\left(z_{1}, z_{2}\right)} \xi \bar{\eta}$, where

$$
\begin{aligned}
\varphi_{0}\left(z_{1}, z_{2}\right) & =\left(z_{1}, z_{2}\right)\left(\begin{array}{cc}
4 & -2 \\
-2 & -2
\end{array}\right) \overline{\left(\begin{array}{l}
z_{1} \\
z_{2}
\end{array}\right)} \\
\varphi_{1}\left(z_{1}, z_{2}\right) & =\left(z_{1}, z_{2}\right)\left(\begin{array}{cc}
2 a+1 & -(a+2) \\
-(a+2) & 2 a+1
\end{array}\right) \overline{\left(\begin{array}{l}
z_{1} \\
z_{2}
\end{array}\right)} \\
\varphi_{2}\left(z_{1}, z_{2}\right) & =\left(z_{1}, z_{2}\right)\left(\begin{array}{cc}
2 a+3 & -a \\
-a & 2 a-3
\end{array}\right) \overline{\left(\begin{array}{l}
z_{1} \\
z_{2}
\end{array}\right)}
\end{aligned}
$$

on each small open subset $U$ of $V$ with appropriate local trivialization $s^{j}$ of $L_{j}$ on $U$. Let us define the variety $X$ as the total space of a $\mathbb{P}^{2}$-bundle $\pi: \mathbb{P}\left(L_{0} \oplus L_{1} \oplus L_{2}\right) \rightarrow V$ over $V$ and $L=\mathcal{O}_{\mathbb{P}\left(L_{0} \oplus L_{1} \oplus L_{2}\right)}(1)$. Let $U$ be a sufficiently small open set of $V$. We use the function

$$
\begin{aligned}
\left(\left[x_{0} ; x_{1} ; x_{2}\right], z_{1}, z_{2}\right) & \mapsto\left[x_{0} s_{0}\left(z_{1}, z_{2}\right) ; x_{1} s_{1}\left(z_{1}, z_{2}\right) ; x_{2} s_{2}\left(z_{1}, z_{2}\right)\right] \\
& \in\left(\mathbb{C} s^{0}\left(z_{1}, z_{2}\right) \oplus \mathbb{C} s^{1}\left(z_{1}, z_{2}\right) \oplus \mathbb{C} s^{2}\left(z_{1}, z_{2}\right)\right)^{*} / \mathbb{C}^{*}=\pi^{-1}\left(z_{1}, z_{2}\right)
\end{aligned}
$$

as a coordinates system on $\pi^{-1}(U)$, where $s_{j}$ is a dual section of $s^{j}$. Using these coordinates, our main result applied to this example can be stated as follows:

TheOrem 1.1. Let $(X, L)$ be the above example, which is introduced by Nakayama [14] and admits no Zariski decomposition even after modifications. There is a minimal singular metric $h_{\min }$ on $L$ whose local weight function $\psi$ is continuous on $X \backslash \mathbb{P}\left(L_{0}\right)$ and is written as

$$
\psi=\log \max _{(\alpha, \beta) \in H}\left(\left|x_{1}\right|^{2 \alpha} \cdot\left|x_{2}\right|^{2 \beta}\right)+O(1)
$$

at each point in $\mathbb{P}\left(L_{0}\right)$ with local coordinates $\left(x_{1}, x_{2}, z_{1}, z_{2}\right)=\left(\left[1 ; x_{1} ; x_{2}\right], z_{1}, z_{2}\right)$, where $H=\left\{(\alpha, \beta) \in \mathbb{R}^{2} \mid \alpha, \beta \geq 0, a^{2}(\alpha+\beta)^{2}=(1-\alpha)^{2}+(1-\beta)^{2}\right\}$.

This expression enables us to compute the multiplier ideal sheaf $\mathcal{J}\left(h_{\text {min }}^{t}\right)$ for each positive number $t$, whose stalk at $x_{0} \in X$ is defined by

$$
\mathcal{J}\left(h_{\text {min }}^{t}\right)_{x_{0}}=\left\{\left.f \in \mathcal{O}_{X, x}|| f\right|^{2} e^{-t \varphi_{\min }} \text { is integrable around } x_{0}\right\},
$$

where $\varphi_{\min }$ is the local weight function of $h_{\min }$ around $x_{0}$.

Corollary 1.2. $\mathcal{J}\left(h_{\text {min }}^{t}\right)$ is trivial at any point in $X \backslash \mathbb{P}\left(L_{0}\right)$. For a point $x_{0} \in \mathbb{P}\left(L_{0}\right)$, the stalk $\mathcal{J}\left(h_{\min }\right)_{x_{0}}$ of the multiplier ideal sheaf is the ideal of $\mathcal{O}_{X, x_{0}}$ which is generated by the polynomials

$$
\left\{x_{1}^{p} x_{2}^{q} \mid(p+1, q+1) \in \operatorname{Int}\left(S_{t}\right) \cap \mathbb{Z}^{2}\right\},
$$

where we denote by $S_{t}$ the set $\left\{(t \alpha, t \beta) \in \mathbb{R}^{2} \mid \alpha, \beta \geq 0, a^{2}(\alpha+\beta)^{2} \geq(1-\alpha)^{2}+(1-\beta)^{2}\right\}$ ( For the shape of $S_{t}$ in this case, see Figure 1). 


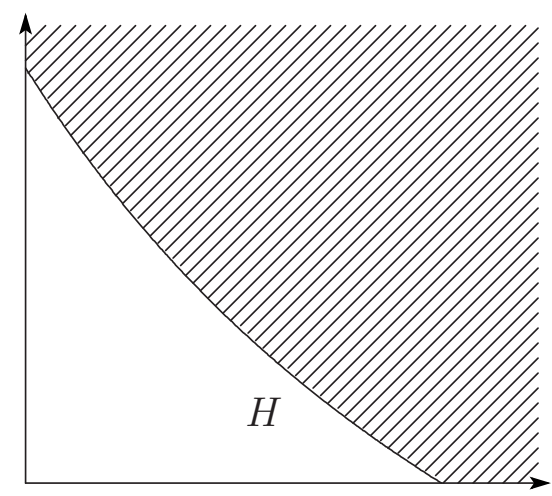

FigurE 1. The shaded area of this figure represents the set $S_{1}$. The set $S_{t}$ is the set of points $p \in \mathbb{R}^{2}$ which satisfies $\frac{p}{t} \in S_{1}$.

According to [14], this $(X, L)$ is an example which admits no Zariski decomposition even after modifications. So, it can be expected in this case that the behavior of this multiplier ideal sheaf is different from the algebraic cases. Indeed, the set of jumping numbers $\operatorname{Jump}\left(\psi ; x_{0}\right)$ for a point $x$ in $\mathbb{P}\left(L_{0}\right)$ (see [9, Section 5] for definition) can be written as follows in this case;

$$
\operatorname{Jump}\left(\psi ; x_{0}\right)=\left\{\frac{p+\sqrt{2 p^{2} a^{2}-q^{2}}}{2} \mid p, q \in \mathbb{Z}, 0 \leq q<p, p-q \equiv 0(\bmod 2)\right\},
$$

which is the set of the largest roots of the quadratic equations $4 T^{2}-4 p T+\left(1-2 a^{2}\right) p^{2}+q^{2}=$ 0 of $T$, where integers $p$ and $q$ satisfy the above conditions. This set has different properties from algebraic multiplier ideal sheaves. For example, it seems difficult to expect the "periodicity" property, and does not have the "rationality" property in this case (For these property, see [9, 1.12] or Remark 6.3 below). Especially, the singularity exponent $c_{x_{0}}(\psi)$, which is the minimum number in the set of all jumping numbers, satisfies

$$
c_{x_{0}}(\psi)=\sqrt{2} a+1
$$

and it is clearly irrational.

More generally, we give a concrete expression of a minimal singular metric on a big line bundle $L$ on the total space of such a toric bundle, see Theorem 4.7. As an application, we discuss Zariski closedness of the non-nef locus $\operatorname{NNef}(L)$ of $L$, see Corollary 5.5.

The organization of the paper is as follows. Let $X$ be the total space of a smooth projective toric bundle over a complex torus, and $L$ be a big line bundle over $X$. In Section 2, we recall some facts and notations related to analysis on $X$ and $L$. In Section 3 , we fix a way to coordinate $X$, and study how modifications of $X$ or zeros of holomorphic sections of $L$ can be treated by using this coordinates system. In Section 4 , we construct a singular hermitian metric $\left\{e^{-\psi_{\sigma}}\right\}$ of $L$ and show it is a minimal singular metric. In Section 5 , we study some properties related to the positivity of $L$, as applications of the result 
in Section 4. Here we introduce how to calculate the Kiselman numbers and the Lelong numbers of minimal singular metrics, and study the non-nef locus of $L$ and multiplier ideal sheaves associated to minimal singular metrics. In Section 6, we introduce three examples for $(X, L)$, all of which is based on the example introduced in [14], and apply our result to them.

Acknowledgment. The author would like to thank his supervisor Prof. Shigeharu Takayama whose enormous support and insightful comments were invaluable during the course of his study. He is very grateful to Prof. Shunsuke Takagi for valuable comments and various suggestion. He also thanks Tomoyuki Hisamoto and Shin-ichi Matsumura who gave him invaluable comments and warm encouragements. He is supported by the Grant-in-Aid for Scientific Research (KAK- ENHI No. 25-2869) and the Grant-in-Aid for JSPS fellows. This work was supported by the Program for Leading Graduate Schools, MEXT, Japan.

\section{PRELIMinaRies to ANALYSis ON TORIC BUNDLES}

2.1. Analysis on compact Kähler manifolds. Let $X$ be a compact Kähler manifold and $L$ be a holomorhic line bundle on $X$. Let $h$ be a singular hermitian metric on $L$. For each local trivialization of $L$ on an open set of $X$, "the inner product" defined by $h$ can be written as $(\xi, \eta)_{z}=e^{-\psi(z)} \xi \bar{\eta}$ where $z$ is a point in the open set, $\xi$ and $\eta$ are points in $\mathbb{C}$, which we regard as the $z$-fiber of $L$, and $\psi$ is a locally integrable function defined on the open set, which we call the local weight of $h$. The local currents written as $d d^{c} \psi$ for the local weight $\psi$ of $h$ glue together to define the curvature current associated to $h$. We denote it by $\Theta_{h}$.

In order to define the minimal singular metric, let us recall how to compare the singularities of plurisubharmonic functions.

Definition 2.1. ([8, 1.4]) Let $\varphi$ and $\psi$ be plurisubharmonic functions defined on a neighborhood of $x \in X$. We write $\psi \prec_{\text {sing }} \varphi$ at $x$ when there exists a positive constant $C$ such that the inequality $e^{-\varphi} \leq C e^{-\psi}$ holds for each point sufficiently near to $x$. We denote $\varphi \sim_{\text {sing }} \psi$ at $x$ if $\varphi \prec_{\text {sing }} \psi$ and $\varphi \succ_{\text {sing }} \psi$ holds at $x$.

By using this notation, we can define the minimal singular metric as follows.

DEFinition 2.2. Let $h_{\text {min }}$ be a singular hermitian metric on $L$ which satisfies $\Theta_{h_{\min }} \geq 0$. We call $h_{\text {min }}$ a minimal singular metric if $\psi \prec_{\text {sing }} \varphi_{\text {min }}$ holds at any point $x \in X$ for all singular hermitian metric $h$ satisfying $\Theta_{h} \geq 0$, where $\varphi_{\min }$ and $\psi$ stand for the local weight functions of $h_{\min }$ and $h$, respectively, with respect to a local trivialization of $L$ around the point $x \in X$.

It is known that there exists a minimal singular metric on every pseudo-effective line bundle. This fact is proved by considering the upper semi-continuous regularization of 
the supremum of the all appropriately normalized $\psi^{6} \mathrm{~s}$, where $\psi$ is as in Definition 2.2 (see [8, 1.5] for details).

Let $L$ be a big line bundle. We denote by $N(L)$ the negative part $\sum_{\Gamma \text { : prime divisor }} \nu\left(\varphi_{\min }, \Gamma\right) \Gamma$ of $L$ in the sense of the divisorial Zariski decomposition [3], where $\varphi_{\min }$ is the local weight of a minimal singular metric on $L$ and $\nu\left(\varphi_{\min }, \Gamma\right)$ is the Lelong number of $\varphi_{\min }$ at the divisor $\Gamma$. We say that $L$ admits a Zariski decomposition if the positive part $P(L):=c_{1}\left(L \otimes \mathcal{O}_{X}(L)\right)$ is nef class. We here remark that this definition of the Zariskidecomposability coincides with Nakayama's algebraic one [14].

2.2. Complex tori. Here, let us recall some fundamental terminologies related to complex tori. Let $\Lambda \subset \mathbb{C}^{d}$ be a lattice. We denote $\mathbb{C}^{d} / \Lambda$ by $V$ and the natural map $\mathbb{C}^{d} \rightarrow V$ by $p$.

Proposition 2.3. ([2, Chapter 3]) Following four propositions hold for above $d, V$, and $\Lambda$ as above. Here, let us denote by $\mathbb{H}_{d}$ the set of all hermitian matrices of size $d \times d$ with $\mathbb{C}$-coefficients.

(1) There exists an injective $\mathbb{R}$-linear map $\mathrm{NS}(V) \otimes \mathbb{R} \rightarrow \mathbb{H}_{d}$.

(2) By this linear map, $\mathrm{NS}(V)$ is identified with $\left\{H \in \mathbb{H}_{d} \mid \forall \lambda, \mu \in \Lambda, \operatorname{Im}(\lambda H \bar{\mu}) \in \mathbb{Z}\right\}$.

(3) By this linear map, the nef cone $\operatorname{Nef}(V) \subset \mathrm{NS}(V)$ is identified with

$$
\left\{H \in \mathbb{H}_{d} \mid H \geq 0 \text { and } H \text { is an element of the image of the set } \mathrm{NS}(V) \otimes \mathbb{R}\right\} \text {. }
$$

(4) Let $c_{1}(E)$ be identified with $H_{E} \in \mathbb{H}_{d}$ by this linear map for a line bundle $E$ on $V$. Fix a metric $h_{E}$ of $E$ whose curvature form is a harmonic form with respect to the Euclidean metric (such $h_{E}$ always exists and is unique up to scale). Here we fix a point of $V$ and denote by $z=\left(z_{1}, z_{2}, \ldots, z_{d}\right)$ the local coordinates of $V$ around the point induced by the map $p$ and the usual coordinates of $\mathbb{C}^{d}$. Then, there exists a canonically determined local frame e of $E$ on the neighborhood of the point such that, with respect to this local trivialization, the local weight function $\varphi_{E}$ of $h_{E}$ can be written as

$$
\varphi_{E}\left(z_{1}, z_{2}, \ldots, z_{d}\right)=\left(z_{1}, z_{2}, \ldots, z_{d}\right) H_{E} \overline{\left(\begin{array}{c}
z_{1} \\
z_{2} \\
\vdots \\
z_{d}
\end{array}\right) .} .
$$

2.3. Toric bundles. Here, we review fundamental terminology related to toric bundles. We follow [14, IV] basically. Let us denote by $V$ a base complex manifold. For simplicity, we restrict ourselves to the case where $V$ is a complex torus. Let $N$ be a free $\mathbb{Z}$-module of rank $n$, and $M$ be the dual module $\operatorname{Hom}(N, \mathbb{Z})$. We denote by $e_{1}, e_{2}, \ldots, e_{n}$ generators of $N$, and by $e^{1}, e^{2}, \ldots, e^{n}$ the dual generators of $M$. We write $N_{\mathbb{R}}$ and $M_{\mathbb{R}}$ for $N \otimes \mathbb{R}$ and $M \otimes \mathbb{R}$, respectively. We fix a group homomorphism

$$
\mathcal{L}: M \rightarrow \operatorname{Pic}(V)
$$


and a fan $\Sigma$ of $N$, and construct a toric bundle $\pi: \mathbb{T}_{N}(\Sigma, \mathcal{L}) \rightarrow V$. We assume the fan $\Sigma$ is smooth projective, which means that the fan is defined by a smooth full-dimensional lattice polytope. Under this assumption, the toric variety $\mathbb{T}_{N}(\Sigma)$ is a smooth projective variety. We denote by $\mathcal{L}^{m} \in \operatorname{Pic}(V)$ the image of $m \in M$. For simplicity, we also denote by $\mathcal{L}^{m}$ the image of $m \in M_{\mathbb{R}}$ with respect to the linear map

$$
\mathcal{L} \otimes \mathbb{R}: M_{\mathbb{R}} \rightarrow \operatorname{Pic}(V) \otimes \mathbb{R} .
$$

Definition 2.4. For $\sigma \in \Sigma$, we define the affine toric bundle $\pi: \mathbb{T}_{N}(\sigma, \mathcal{L}) \rightarrow V$ by

$$
\mathbb{T}_{N}(\sigma, \mathcal{L})=\operatorname{Spec}_{V} \bigoplus_{m \in \sigma^{\vee} \cap M} \mathcal{L}^{m}
$$

with the canonical morphism to $V$, and the toric bundle $\pi: \mathbb{T}_{N}(\Sigma, \mathcal{L}) \rightarrow V$ by gluing $\left\{\mathbb{T}_{N}(\sigma, \mathcal{L}) \rightarrow V\right\}_{\sigma \in \Sigma}$ in the natural way.

For each cone $\sigma \in \Sigma$, there exists a corresponding $\mathbb{T}:=\operatorname{Hom}\left(M, \mathbb{C}^{*}\right)$-orbit $\mathbb{O}_{\sigma}(\mathcal{L})$ as the case of toric varieties. Let us denote by $\mathbb{V}(\sigma, \mathcal{L})$ the closure of $\mathbb{O}_{\sigma}(\mathcal{L})$ as the subset of $\mathbb{T}_{N}(\Sigma, \mathcal{L})$. Just as the case of toric varieties, the codimension of $\mathbb{V}(\sigma, \mathcal{L})$ coincides with the dimension of $\sigma$. In particular, for each 1-dimensional $\sigma \in \Sigma, \mathbb{V}(\sigma, \mathcal{L})$ is a prime divisor of $\mathbb{T}_{N}(\Sigma, \mathcal{L})$.

Definition 2.5. We denote by $\operatorname{Ver}(\Sigma)$ the set of the whole primitive generators $v \in N$ of one-dimensional cones of $\Sigma$. For $v \in \operatorname{Ver}(\Sigma)$, we denote by $\Gamma_{v}$ the prime divisor $\mathbb{V}\left(\mathbb{R}_{\geq 0} v, \mathcal{L}\right)$. Let us set

$$
\mathrm{PL}_{N}(\Sigma, \mathbb{Z})=\left\{h: N_{\mathbb{R}} \rightarrow \mathbb{R} \mid \text { for each } \sigma \in \Sigma,\left.h\right|_{\sigma} \text { is linear, and } h(N) \subset \mathbb{Z}\right\} .
$$

For $h \in \mathrm{PL}_{N}(\Sigma, \mathbb{Z})$, we define the divisor $D_{h}$ by

$$
D_{h}=\sum_{v \in \operatorname{Ver}(\Sigma)}(-h(v)) \Gamma_{v}
$$

It is known that any line bundle over $\mathbb{T}_{N}(\Sigma, \mathcal{L})$ can be written by adding a divisor of the form $D_{g}$ to the pull-back of a line bundle over $V([14,2.3])$.

ExAmple 2.6. The cone $\{0\}$ is always an element of the fan $\Sigma$. Here we consider the affine toric bundle $\mathbb{T}_{N}(\{0\}, \mathcal{L})$. Fix a metric on $\mathcal{L}^{e^{j}}$ whose curvature form is a harmonic form with respect to the Euclidean metric for each $j$. Let $U$ be a sufficiently small open set in $V$ and $z \mapsto s^{j}(z)$ be such a local trivialization of $\mathcal{L}^{e^{j}}$ on $U$ as in Proposition 2.3, and $z \mapsto s_{j}(z)$ be the dual frame of the local frame $z \mapsto s^{j}(z)$ for $j=1,2, \ldots, n$. It can be easily checked that the frame $z \mapsto s_{j}(z)$ is also such a section of $\mathcal{L}^{-e^{j}}=\left(\mathcal{L}^{e^{j}}\right)^{-1}$ as in Proposition 2.3. Here,

$$
\begin{aligned}
\left.\mathbb{T}_{N}(\{0\}, \mathcal{L})\right|_{\{z\}} & =\operatorname{Spec} \mathbb{C}\left[s^{1}(z), s^{2}(z), \ldots, s^{n}(z),\left(s^{1}\right)^{-1}(z),\left(s^{2}\right)^{-1}(z), \ldots,\left(s^{n}\right)^{-1}(z)\right] \\
& =\prod_{j=1}^{n} \mathbb{C}^{*} \cdot s_{j}(z)
\end{aligned}
$$


for $z \in U$. Thus, it follows that the affine toric bundle $\mathbb{T}_{N}(\{0\}, \mathcal{L})$ can be considered as the $\left(\mathbb{C}^{*}\right)^{n}$-bundle on $V$ of which the system $\left\{s_{j}\right\}_{j}$ works as a local trivialization on $U$.

ExAmple 2.7. Second example is a case where $n=2$. Let $L_{0}, L_{1}, L_{2}$ be line bundles over $V$. Let $\mathcal{L}$ be a map defined by $e^{j} \mapsto L_{j} \otimes L_{0}^{-1}(j=1,2)$ and $\Sigma$ be the fan generated by the three cones

$$
\sigma_{1}=\text { Cone }\left\{e_{1}, e_{2}\right\}, \sigma_{2}=\operatorname{Cone}\left\{e_{2},-\left(e_{1}+e_{2}\right)\right\}, \text { and } \sigma_{3}=\operatorname{Cone}\left\{-\left(e_{1}+e_{2}\right), e_{1}\right\} .
$$

Fix a metric on $\mathcal{L}^{e^{j}}$ whose curvature form is a harmonic form with respect to the Euclidean

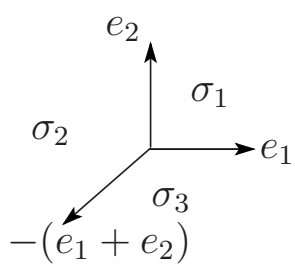

Figure 2. $\Sigma$.

metric for each $j$. Let $U$ be a sufficiently small open set in $V$ and $z \mapsto s_{1}(z), z \mapsto s_{2}(z)$ be such local trivializations of $\left(L_{1} \otimes L_{0}^{-1}\right)^{-1},\left(L_{2} \otimes L_{0}^{-1}\right)^{-1}$ of $U$ as in Proposition 2.3, respectively, and $s^{j}$ be the dual of $s_{j}$ for $j=1,2$. Here,

$$
\begin{aligned}
\left.\mathbb{T}_{N}\left(\sigma_{1}, \mathcal{L}\right)\right|_{\{z\}} & =\operatorname{Spec} \mathbb{C}\left[s^{1}(z), s^{2}(z)\right], \\
\left.\mathbb{T}_{N}\left(\sigma_{2}, \mathcal{L}\right)\right|_{\{z\}} & =\operatorname{Spec} \mathbb{C}\left[\left(s^{1}(z)\right)^{-1} s^{2}(z),\left(s^{1}(z)\right)^{-1}\right], \\
\left.\mathbb{T}_{N}\left(\sigma_{3}, \mathcal{L}\right)\right|_{\{z\}} & =\operatorname{Spec} \mathbb{C}\left[\left(s^{2}(z)\right)^{-1}, s^{1}(z)\left(s^{2}(z)\right)^{-1}\right],
\end{aligned}
$$

for $z \in U$. Using this expressions, we can calculate that

$$
\mathbb{T}_{N}(\Sigma, \mathcal{L})=\mathbb{P}\left(\mathcal{O}_{V} \oplus\left(L_{1} \otimes L_{0}^{-1}\right) \oplus\left(L_{2} \otimes L_{0}^{-1}\right)\right) \cong \mathbb{P}\left(L_{0} \oplus L_{1} \oplus L_{2}\right) .
$$

In this case, $\operatorname{Ver}(\Sigma)$ is the set consisting of the following three elements; $v_{0}=-\left(e_{1}+\right.$ $\left.e_{2}\right), v_{1}=e_{1}$, and $v_{2}=e_{2}$. Let us define $h \in \mathrm{PL}_{N}(\Sigma, \mathbb{Z})$ by $v_{0} \mapsto-1, v_{1} \mapsto 0$, and $v_{2} \mapsto 0$. Then the line bundle $L=\mathcal{O}_{\mathbb{P}\left(L_{0} \oplus L_{1} \oplus L_{2}\right)}(1)$ can be written as

$$
L \cong \pi^{*} L_{0} \otimes \mathcal{O}_{X}\left(D_{h}\right)
$$

\section{TORIC BUNDLES OVER COMPLEX TORI}

3.1. Holomorphic sections and local coordinates. Let $V$ be a smooth projective variety and $\Sigma$ be the fan defined by a smooth full-dimensional lattice polytope of $M$ just as in the previous section. We denote by $X$ the total space of the toric bundle $\pi: \mathbb{T}_{N}(\Sigma, \mathcal{L}) \rightarrow V$. Here we consider holomorphic sections of a line bundle $L$ over $X$. According to $([14,2.3])$, without loss of generality, we may assume $L=\pi^{*} L_{0} \otimes \mathcal{O}_{X}\left(D_{h}\right)$, where $L_{0}$ is a holomorphic line bundle over $V$, and $h$ is an element of $\operatorname{PL}_{N}(\Sigma, \mathbb{Z})$. 
Definition 3.1. We denote by $\square_{h}$ the set $\left\{m \in M_{\mathbb{R}} \mid \forall x \in N_{\mathbb{R}},\langle m, x\rangle \geq h(x)\right\}$, and by $\square_{\text {Nef }}\left(L_{0}, h\right)$ the set $\left\{m \in \square_{h} \mid L_{0} \otimes \mathcal{L}^{m}\right.$ is nef $\}$ for a line bundle $L_{0}$ over $V$ and an element $h \in \mathrm{PL}_{N}(\Sigma, \mathbb{Z})$.

Since $\square_{h}$ is a bounded closed convex set, we clearly obtain the following lemma.

LEMMA 3.2. $\square_{\mathrm{Nef}}\left(L_{0}, h\right)$ is a bounded closed convex subset of $M_{\mathbb{R}}$.

Definition 3.3. Here we use notations in Example 2.6. For $m \in M$, we define the meromorphic section $\chi^{m}$ of $\pi^{*} \mathcal{L}^{-m}$ on $\mathbb{T}_{N}(\Sigma, \mathcal{L})$ by

$$
\left(x_{j} \cdot s_{j}(z)\right)_{j} \longmapsto \prod_{j=1}^{n}\left(x_{j} \cdot s_{j}(z)\right)^{m_{j}}=\left(x_{1}\right)^{m_{1}} \cdot\left(x_{2}\right)^{m_{2}} \cdots\left(x_{n}\right)^{m_{n}} \cdot\left(\prod_{j=1}^{n}\left(s^{j}\right)^{-m_{j}}\right)(z)
$$

on $\left.\mathbb{T}_{N}(\{0\}, \mathcal{L})\right|_{U}$, where $m_{j}=\left\langle m, e_{j}\right\rangle$.

$\mathbb{T}_{N}(\{0\}, \mathcal{L})$, which we considered in Example 2.6, is always a dense subset of $\mathbb{T}_{N}(\Sigma, \mathcal{L})$. In the case of toric varieties, or the case that $V$ is the " 0 -dimensional complex torus", regular functions on $\mathbb{T}_{N}(\Sigma, \mathcal{L})$ can be regarded as meromorphic functions on $\mathbb{T}_{N}(\{0\}, \mathcal{L})$. There is an analogue of this fact in the general setting.

Proposition 3.4. ([14, 2.3, 2.4]) The line bundle $L=\pi^{*} L_{0} \otimes \mathcal{O}_{X}\left(D_{h}\right)$ is pseudoeffective if and only if the set $\square_{\mathrm{Nef}}\left(L_{0}, h\right)$ is non-empty. In this case, we obtain the equation

$$
H^{0}(X, L)=\bigoplus_{m \in \square_{\mathrm{Nef}}\left(L_{0}, h\right) \cap M} \chi^{m} \cdot \pi^{*} H^{0}\left(V, L_{0} \otimes \mathcal{L}^{m}\right) .
$$

In the following, we assume that $V$ is a complex torus.

Observation 3.5. Here we rewrite the meromorphic function $\chi^{m} \cdot \pi^{*} f$ in Proposition 3.4 by using notations in Example 2.6. Let $U$ be a sufficiently small open set in $V$ and $z \mapsto s^{0}(z)$ be such a local trivialization of $L_{0}$ on $U$ as in Proposition 2.3. Under the local trivialization $z \mapsto\left(s^{0} \cdot \prod_{j=1}^{n} s^{j}\right)(z)$ of $L_{0} \otimes \mathcal{L}^{m}$, we may assume $f$ is written as

$$
\left.f\right|_{U}(z)=\eta(z) \cdot\left(s^{0} \cdot \prod_{j=1}^{n}\left(s^{j}\right)^{\left\langle m, e_{j}\right\rangle}\right)(z)
$$

on $U$ for some holomorphic function $\eta$ on $U$. Since

$$
\chi^{m} \cdot \pi^{*} f\left(\left(x_{j} \cdot s_{j}(z)\right)_{j}\right)=\chi^{m}\left(\left(x_{j} \cdot s_{j}(z)\right)_{j}\right) \cdot f(z)=\left(\prod_{j=1}^{n}\left(x_{j}\right)^{\left\langle m, e_{j}\right\rangle}\right) \eta(z) \cdot s^{0}(z)
$$

holds, it can be checked that $\chi^{m} \cdot \pi^{*} f$ is a meromorphic section of $\pi^{*} L_{0}$, indeed. Moreover we can check that it is an element of $H^{0}(X, L)=H^{0}\left(X, \pi^{*} L_{0} \otimes \mathcal{O}_{X}\left(D_{h}\right)\right)$, since $m$ is an element of $\square_{h}$. 
In Observation 3.5, we calculated $\chi^{m} \cdot \pi^{*} f$ as a meromorphic section of $\pi^{*} L_{0}$. We can rewrite it as a holomorphic section of $\pi^{*} L_{0} \otimes \mathcal{O}_{X}\left(D_{h}\right)$ by using following canonical local coordinates.

Definition 3.6. Let $\sigma$ be an element of $\Sigma_{\max }:=\{\sigma \in \Sigma \mid \operatorname{dim} \sigma=n\}$. Since the fan $\Sigma$ is smooth, there exists $v_{1}, v_{2}, \ldots, v_{n} \in \operatorname{Ver}(\Sigma)$ such that $\sigma=\operatorname{Cone}\left\{v_{1}, v_{2}, \ldots, v_{n}\right\}$ and $v_{1}, v_{2}, \ldots, v_{n}$ generates $N$. We call such $v_{1}, v_{2}, \ldots, v_{n} N$-minimal generators of $\sigma$.

Let $v^{1}, v^{2}, \ldots, v^{n}$ be the dual generators of $v_{1}, v_{2}, \ldots, v_{n}$. Then the dual cone of $\sigma$ can be written as $\sigma^{\vee}=$ Cone $\left\{v^{1}, v^{2}, \ldots, v^{n}\right\}$. Fix a metric $h_{v^{j}}$ of $\mathcal{L}^{v^{j}}$ whose curvature form is a harmonic form with respect to the Euclidean metric for each $j$. Let $U$ be a sufficiently small open set in $V$. Let us fix such a local trivializations $z \mapsto t^{j}(z)$ of $\mathcal{L}^{v^{j}}$ on $U$ as in Proposition 2.3, and the dual section $t_{j}$ of $t^{j}$ for $j=1,2, \ldots, n$. Using these notations, we can calculate

$$
\left.\mathbb{T}_{N}(\sigma, \mathcal{L})\right|_{\{z\}}=\left.\operatorname{Spec} \bigoplus_{a_{1}, a_{2}, \ldots, a_{n} \geq 0} \mathcal{L}^{\sum_{j} a_{j} v^{j}}\right|_{\{z\}}=\operatorname{Spec} \mathbb{C}\left[t^{1}(z), t^{2}(z), \ldots, t^{n}(z)\right]
$$

for $z \in U$. So, it turns out that $\mathbb{T}_{N}(\sigma, \mathcal{L})$ is a $\mathbb{C}^{n}$-bundle which $t_{1}, t_{2}, \ldots, t_{n}$ gives a local trivialization on $U$. So, we can regard the map

$$
\left.\left(x_{1}, x_{2}, \ldots, x_{n}, z\right) \longmapsto\left(x_{j} \cdot t_{j}(z)\right)_{j} \in \mathbb{T}_{N}(\sigma, \mathcal{L})\right|_{\{z\}}
$$

as a local coordinates system on $\left.\mathbb{T}_{N}(\sigma, \mathcal{L})\right|_{U}$. We call this local coordinate system the canonical one of $\left.\mathbb{T}_{N}(\sigma, \mathcal{L})\right|_{U}$ associated to the $N$-minimal generator $v_{1}, v_{2}, \ldots, v_{n}$ of $\sigma$.

As it is clear from the definition, the canonical coordinates system of $\left.\mathbb{T}_{N}(\sigma, \mathcal{L})\right|_{U}$ associated to the $N$-minimal generator $v_{1}, v_{2}, \ldots, v_{n}$ of $\sigma$ depends on the choice of the metrics $\left\{h_{v^{j}}\right\}_{j}$. In the following, we fix basis $e^{1}, e^{2}, \ldots, e^{n}$ of $M$ and a metric $h_{e^{j}}$ of $\mathcal{L}^{e^{j}}$ whose curvature form is a harmonic form with respect to the Euclidean metric for each $j$, and we always choose the metric $h_{e^{1}}^{\otimes a_{1}^{j}} \otimes h_{e^{2}}^{\otimes a_{2}^{j}} \otimes \cdots \otimes h_{e^{n}}^{\otimes a_{n}^{j}}$ for $h_{v^{j}}$, where $v^{j}=\sum_{k} a_{k}^{j} e^{k}$. By using this metric, we can say that the canonical coordinates system of $\left.\mathbb{T}_{N}(\sigma, \mathcal{L})\right|_{U}$ associated to the $N$-minimal generator $v_{1}, v_{2}, \ldots, v_{n}$ is uniquely determined.

REMARK 3.7. Let $v_{1}, v_{2}, \ldots, v_{n}$ be $N$-minimal generators of $\sigma$, and $\left(x_{1}, x_{2}, \ldots, x_{n}, z\right)$ be the canonical coordinates system of $\left.\mathbb{T}_{N}(\sigma, \mathcal{L})\right|_{U}$ associated to $v_{1}, v_{2}, \ldots, v_{n}$. Then, $\left\{x_{j}=0\right\}=\Gamma_{v_{j}}$ holds for $j=1,2, \ldots, n$ on $\left.\mathbb{T}_{N}(\sigma, \mathcal{L})\right|_{U}$.

Definition 3.8. For $\sigma \in \Sigma_{\max }$, we denote by $m_{\sigma} \in M$ the point which satisfies $h(w)=\left\langle m_{\sigma}, w\right\rangle$ for all $w \in \sigma$. We call $\left\{m_{\sigma}\right\}_{\sigma}$ the Cartier data of $D_{h}$.

OBSERVATION 3.9. Let $\sigma$ be an element of $\Sigma_{\max }, v_{1}, v_{2}, \ldots, v_{n}$ be $N$-minimal generators of $\sigma$, and $\left(x_{1}, x_{2}, \ldots, x_{n}, z\right)$ be the canonical coordinates system of $\left.\mathbb{T}_{N}(\sigma, \mathcal{L})\right|_{U}$ associated 
to $v_{1}, v_{2}, \ldots, v_{n}$. In $\left.\mathbb{T}_{N}(\sigma, \mathcal{L})\right|_{U}$, the map

$$
\left(x_{1}, x_{2}, \ldots, x_{n}, z\right) \mapsto \prod_{j=1}^{n}\left(x_{j}\right)^{\left\langle m_{\sigma}, v_{j}\right\rangle}
$$

gives a local trivialization of $\mathcal{O}_{X}\left(D_{h}\right)$, where $\left\{m_{\sigma}\right\}_{\sigma}$ is the Cartier data of $D_{h}$. So, by using notations in Observation 3.5 ,

$$
\left(x_{1}, x_{2}, \ldots, x_{n}, z\right) \longmapsto\left(\prod_{j=1}^{n}\left(x_{j}\right)^{\left\langle m_{\sigma}, v_{j}\right\rangle}\right) \cdot s^{0}(z)
$$

gives a local trivialization of $L$. Under this trivialization, $\chi^{m} \cdot \pi^{*} f \in H^{0}(X, L)$ can be regarded as the holomorphic function

$$
\left(x_{1}, x_{2}, \ldots, x_{n}, z\right) \longmapsto\left(\prod_{j=1}^{n}\left(x_{j}\right)^{\left\langle m-m_{\sigma}, v_{j}\right\rangle}\right) \cdot \eta(z)
$$

on $\left.\mathbb{T}_{N}(\sigma, \mathcal{L})\right|_{U}$

The projective line $\mathbb{P}^{1}=\{[z ; w]\}$ can be regarded as the union of two disks $\{[z ; 1] \mid$ $|z| \leq 1\}$ and $\{[1 ; w]|| w \mid \leq 1\}$ with radius 1 . The following proposition is an analogy of this fact.

Proposition 3.10. Let $U$ be a sufficiently small open set in $V$, $z_{0}$ be a point in $U, \sigma$ be an element of $\Sigma_{\max }, v_{1}, v_{2}, \ldots, v_{n}$ be $N$-minimal generators of $\sigma$, and $\left(x_{1}, x_{2}, \ldots, x_{n}, z\right)$ be the canonical coordinates system of $\left.\mathbb{T}_{N}(\sigma, \mathcal{L})\right|_{U}$ associated to $v_{1}, v_{2}, \ldots, v_{n}$. We set

$$
K_{\sigma, z_{0}}=\left\{\left(x_{1}, x_{2}, \ldots, x_{n}, z_{0}\right) \in \mathbb{T}_{N}(\sigma, \mathcal{L})|\forall j \in\{1,2, \ldots, n\},| x_{j} \mid \leq 1\right\} .
$$

Then,

$$
\bigcup_{\sigma \in \Sigma_{\max }} K_{\sigma, z_{0}}=\pi^{-1}\left(z_{0}\right)
$$

holds.

ProOF. Since $\overline{\left.\mathbb{T}_{N}(\{0\}, \mathcal{L})\right|_{\left\{z_{0}\right\}}}=\pi^{-1}\left(z_{0}\right)$, it is sufficient to show that

$$
\left.\bigcup_{\sigma \in \Sigma_{\max }} K_{\sigma, z_{0}} \supset \mathbb{T}_{N}(\{0\}, \mathcal{L})\right|_{\left\{z_{0}\right\}}
$$

Let us fix a point $\left.y_{0} \in \mathbb{T}_{N}(\{0\}, \mathcal{L})\right|_{\left\{z_{0}\right\}}$ and an element $\tau \in \Sigma_{\max }$. Let $u_{1}, u_{2}, \ldots, u_{n}$ be $N$-minimal generators of $\tau$, and $\left(y_{1}, y_{2}, \ldots, y_{n}, z\right)$ be the canonical coordinates system of $\left.\mathbb{T}_{N}(\tau, \mathcal{L})\right|_{U}$ associated to $u_{1}, u_{2}, \ldots, u_{n}$. In this coordinates system, assume $y_{0}$ is written as $\left(\left(y_{0}\right)_{1},\left(y_{0}\right)_{2}, \ldots,\left(y_{0}\right)_{n}, z_{0}\right)$. Since $y_{0} \in \mathbb{T}_{N}(\{0\}, \mathcal{L})$, it turns out that $\left(y_{0}\right)_{j} \neq 0$ for all $j$. Thus, $w_{0}=-\sum_{j=1}^{n} \log \left|\left(y_{0}\right)_{j}\right| \cdot u_{j}$ defines a point of $N_{\mathbb{R}}$. Since $\Sigma$ is complete, there exists an element $\sigma \in \Sigma_{\max }$ such that $n_{0} \in \sigma$. Let $v_{1}, v_{2}, \ldots, v_{n}$ be $N$-minimal generators 
of $\sigma$, and $\left(x_{1}, x_{2}, \ldots, x_{n}, z\right)$ be the canonical coordinates system of $\left.\mathbb{T}_{N}(\sigma, \mathcal{L})\right|_{U}$ associated to $v_{1}, v_{2}, \ldots, v_{n}$. In this coordinates system, $y_{0}$ can be written as

$$
y_{0}=\left(\left(\prod_{k=1}^{n}\left(\left(y_{0}\right)_{k}\right)^{\left\langle v^{j}, u_{k}\right\rangle}\right)_{j}, z_{0}\right)
$$

where $v^{1}, v^{2}, \ldots, v^{n}$ is the dual basis of $v_{1}, v_{2}, \ldots, v_{n}$. On the other hands, $w_{0}$ can be rewritten as

$w_{0}=-\sum_{k=1}^{n} \log \left|\left(y_{0}\right)_{k}\right| \cdot u_{k}=-\sum_{k=1}^{n} \sum_{j=1}^{n} \log \left|\left(y_{0}\right)_{k}\right|\left\langle v^{j}, u_{k}\right\rangle \cdot v_{j}=-\sum_{j=1}^{n} \log \left|\prod_{k=1}^{n}\left(\left(y_{0}\right)_{k}\right)^{\left\langle v^{j}, u_{k}\right\rangle}\right| \cdot v_{j}$.

Since we have chosen $\sigma$ as the condition $n_{0} \in \sigma$ holds, $-\log \left|\prod_{k=1}^{n}\left(\left(y_{0}\right)_{k}\right)^{\left\langle v^{j}, u_{k}\right\rangle}\right| \geq 0$, or $\left|\prod_{k=1}^{n}\left(\left(y_{0}\right)_{k}\right)^{\left\langle v^{j}, u_{k}\right\rangle}\right| \leq 1$ holds for all $j \in\{1,2, \ldots, n\}$. We thus obtain $y_{0} \in K_{\sigma, z_{0}}$, which proves the proposition.

3.2. Modifications. Let $\Sigma$ be a smooth projective fan of the $n$-dimensional lattice $N$. Here we fix a smooth subdivision fan $\tilde{\Sigma}$ of $\Sigma$, and consider a toric bundle $\tilde{X}=\mathbb{T}_{N}(\tilde{\Sigma}, \mathcal{L})$ and the canonical morphism $\mu: \tilde{X} \rightarrow X$. As in the case of toric varieties, $\mu: \tilde{X} \rightarrow X$ is a proper modification of $X$. From this section, we use letters with subscripts such as $v_{1}, v_{2}, \ldots, v_{n}$ for generators of $N$, and we denote the dual generators by the same letters with superscripts, such as $v^{1}, v^{2}, \ldots, v^{n}$, throughout this paper.

First of all, we obtain the following result by simple computations.

LEMMA 3.11. Let $\sigma \in \Sigma_{\max }, \tilde{\sigma} \in \tilde{\Sigma}_{\max }$ be cones such that $\tilde{\sigma} \subset \sigma, v_{1}, v_{2}, \ldots, v_{n}$ be $N$ minimal generators of $\sigma$, and $\tilde{v}_{1}, \tilde{v}_{2}, \ldots, \tilde{v}_{n}$ be $N$-minimal generators of $\tilde{\sigma}$. We denote by $\left(x_{1}, x_{2}, \ldots, x_{n}, z\right)$ and $\left(\tilde{x}_{1}, \tilde{x}_{2}, \ldots, \tilde{x}_{n}, z\right)$ the canonical coordinates systems of $\left.\mathbb{T}_{N}(\sigma, \mathcal{L})\right|_{U}$ and $\left.\mathbb{T}_{N}(\tilde{\sigma}, \mathcal{L})\right|_{U}$, respectively. In these coordinates, the morphism $\mu: \tilde{X} \rightarrow X$ can be written as

$$
\mu\left(\tilde{x}_{1}, \tilde{x}_{2}, \ldots, \tilde{x}_{n}, z\right)=\left(\left(\prod_{k=1}^{n}\left(\tilde{x}_{k}\right)^{\left\langle v^{j}, \tilde{v}_{k}\right\rangle}\right)_{j}, z\right)
$$

Lemma 3.11 immediately implies the following corollary.

Corollary 3.12. For $j \in\{1,2, \ldots, n\}$, there exists a subset $J_{v_{j}} \subset\{1,2, \ldots, n\}$ such that $\mu^{*} \Gamma_{v_{j}}=\bigcup_{k \in J_{v_{j}}}\left\{\tilde{x}_{k}=0\right\}$ in $\left.\mathbb{T}_{N}(\tilde{\sigma}, \mathcal{L})\right|_{U}$.

REMARK 3.13. For Corollary 3.12 , the set $J_{v_{j}}$ can be written as

$$
J_{v_{j}}=\left\{k \in\{1,2, \ldots, n\} \mid\left\langle v^{j}, \tilde{v}_{k}\right\rangle \neq 0\right\} .
$$

For $\sigma \in \Sigma_{\max }$, we define the set $\tilde{\Sigma}_{\sigma}$ by $\tilde{\Sigma}_{\sigma}:=\{\tilde{\sigma} \in \tilde{\Sigma} \mid \tilde{\sigma} \subset \sigma\}$, and we denote by $\left(\tilde{\Sigma}_{\sigma}\right)_{\max }$ the set $\left\{\tilde{\sigma} \in \tilde{\Sigma}_{\sigma} \mid \operatorname{dim} \tilde{\sigma}=n\right\}$. By using the expression of $\mu$ in Lemma 3.11, we can get the following lemma. 
Lemma 3.14. Fix a point $z_{0} \in U$, a set $I \subset\{1,2, \ldots, n\}$, and a cone $\sigma \in \Sigma_{\max }$. Denote by $W_{I, \sigma, z_{0}}$ the set

$$
\left\{\left(x_{1}, x_{2}, \ldots, x_{n}, z_{0}\right) \in \mathbb{T}_{N}(\sigma, \mathcal{L})|\forall j \in I,| x_{j} \mid \leq 1, \forall j \in\{1,2, \ldots, n\}, x_{j} \neq 0\right\},
$$

and by $W_{I, \tilde{\sigma}, z_{0}}$ the set

$$
\left\{\left(\tilde{x}_{1}, \tilde{x}_{2}, \ldots, \tilde{x}_{n}, z_{0}\right) \in \mathbb{T}_{N}(\tilde{\sigma}, \mathcal{L})\left|\forall k \in \cup_{j \in I} J_{v_{j}},\right| \tilde{x}_{k} \mid \leq 1, \forall j \in\{1,2, \ldots, n\}, \tilde{x}_{j} \neq 0\right\}
$$

for each $\tilde{\sigma} \in\left(\tilde{\Sigma}_{\sigma}\right)_{\max }$. Then,

$$
\mu\left(\bigcup_{\tilde{\sigma} \in\left(\tilde{\Sigma}_{\sigma}\right)_{\max }} W_{I, \tilde{\sigma}, z_{0}}\right)=W_{I, \sigma, z_{0}}
$$

holds.

This lemma can be proved in the almost same way as those used in Lemma 3.10. Applying this lemma with $I=\{1,2, \ldots, n\}$, we obtain the next corollary.

Corollary 3.15. Here we use notations in Lemma 3.14. Denote by $K_{\sigma}$ the set

$$
\left\{\left.\left(x_{1}, x_{2}, \ldots, x_{n}, z\right) \in \mathbb{T}_{N}(\sigma, \mathcal{L})\right|_{\bar{U}}|\forall j \in\{1,2, \ldots, n\},| x_{j} \mid \leq 1\right\}
$$

and by $K_{\tilde{\sigma}}$ the set

$$
\left\{\left.\left(\tilde{x}_{1}, \tilde{x}_{2}, \ldots, \tilde{x}_{n}, z\right) \in \mathbb{T}_{N}(\tilde{\sigma}, \mathcal{L})\right|_{\bar{U}}|\forall j \in\{1,2, \ldots, n\},| \tilde{x}_{j} \mid \leq 1\right\}
$$

for each $n$-dimensional cone $\tilde{\sigma} \in \tilde{\Sigma}_{\sigma}$. Then,

$$
\mu\left(\bigcup_{\tilde{\sigma} \in\left(\tilde{\Sigma}_{\sigma}\right)_{\max }} K_{\tilde{\sigma}}\right)=K_{\sigma}
$$

holds.

3.3. Convex subsets of $M$. Let $\Sigma$ be a smooth projective fan of the $n$-dimensional lattice $N, \sigma \in \Sigma$ be a $n$-dimensional cone, $v_{1}, v_{2}, \ldots, v_{n}$ be $N$-minimal generators of $\sigma$, and $\left(x_{1}, x_{2}, \ldots, x_{n}, z\right)$ be the canonical coordinates system of $\left.\mathbb{T}_{N}(\sigma, \mathcal{L})\right|_{U}$ associated to $v_{1}, v_{2}, \ldots, v_{n}$, where $U$ is a sufficiently small open set in $V$.

Definition 3.16. For $A \subset \sigma^{\vee}$, we denote by $\overline{\bar{A}}$ the set

$$
\left\{m \in \sigma^{\vee} \mid \forall w \in \sigma, \min _{m^{\prime} \in A}\left\langle m^{\prime}, w\right\rangle \leq\langle m, w\rangle\right\} .
$$

When $A=\emptyset$, we formally regards $\overline{\bar{\emptyset}}$ as $\sigma^{\vee}$.

Definition 3.17. Let $m_{\sigma}$ be an element of the Cartier data $D_{h}$ which is associated to $\sigma$. We denote by $S\left(L_{0}, h\right)_{\sigma}$ the subset $\overline{\overline{\left\{m-m_{\sigma} \mid m \in \square_{\mathrm{Nef}}\left(L_{0}, h\right)\right\}}} \subset \sigma^{\vee}$. 
REMARK 3.18. In $\prod_{j \in I}\left\{\left|x_{j}\right|<1\right\} \times \prod_{j \notin I}\left\{x_{j} \in \mathbb{C}\right\} \times U$,

$$
\max _{m \in S\left(L_{0}, h\right)_{\sigma}} \prod_{j \in I}\left|x_{j}\right|^{2\left\langle m, v_{j}\right\rangle}=\max _{m \in \square_{\mathrm{Nef}}\left(L_{0}, h\right)} \prod_{j \in I}\left|x_{j}\right|^{2\left\langle m-m_{\sigma}, v_{j}\right\rangle}
$$

for any $I \subset\{1,2, \ldots, n\}$, where $m_{\sigma}$ is an element of the Cartier data $D_{h}$ which is associated to $\sigma$.

Definition 3.19. For a point $\left.\left(\left(x_{0}\right)_{1},\left(x_{0}\right)_{2}, \ldots,\left(x_{0}\right)_{n}, z_{0}\right) \in \mathbb{T}_{N}(\sigma, \mathcal{L})\right|_{U}$, let us denote by $I$ the set $\left\{j \in\{1,2, \ldots, n\} \mid x_{0}^{j}=0\right\}$. We define the set $P\left(f_{1}, f_{2}, \ldots, f_{l}\right)_{\left(\left(x_{0}\right)_{1},\left(x_{0}\right)_{2}, \ldots,\left(x_{0}\right)_{n}, z_{0}\right)}$ for $f_{1}, f_{2}, \ldots, f_{l} \in \mathcal{O}_{\left(\left(x_{0}\right)_{1},\left(x_{0}\right)_{2}, \ldots,\left(x_{0}\right)_{n}, z_{0}\right)}$ as follows. Let

$$
f_{\nu}\left(x_{1}, x_{2}, \ldots, x_{n}\right)=\sum_{\alpha \geq 0}\left(x_{I}\right)^{\alpha} A_{\nu, \alpha}\left(x_{I^{c}}, z\right),
$$

be the Taylor expansion of each $f_{\nu}(\nu=1,2, \ldots, l)$ around the point $\left(\left(x_{0}\right)_{1},\left(x_{0}\right)_{2}, \ldots,\left(x_{0}\right)_{n}, z_{0}\right)$ for variables $\left\{x_{j}\right\}_{j \in I}$, where $\alpha=\left(a_{j}\right)_{j \in I}$ is a multi-index, the signature " $\left(x_{I}\right)^{\alpha}$ " stands for $\prod_{j \in I}\left(x_{j}\right)^{a_{j}}$, and $A_{\nu, \alpha}$ is the germ of a holomorphic function with $(n-\# I+d)$-variables $\left(x_{I^{c}}, z\right)=\left(\left(x_{j}\right)_{j \notin I}, z\right)$. We define $P\left(f_{1}, f_{2}, \ldots, f_{l}\right)_{\left(\left(x_{0}\right)_{1},\left(x_{0}\right)_{2}, \ldots,\left(x_{0}\right)_{n}, z_{0}\right)}$ by

$$
P\left(f_{1}, f_{2}, \ldots, f_{l}\right)_{\left(\left(x_{0}\right)_{1},\left(x_{0}\right)_{2}, \ldots,\left(x_{0}\right)_{n}, z_{0}\right)}=\overline{\overline{\bigcup_{\nu=1}^{l}\left\{\sum_{j \in I} a_{j} \cdot v^{j} \mid A_{\nu,\left(a_{j}\right)_{j}} \not \equiv 0\right\}}} \subset \sigma^{\vee} .
$$

Remark 3.20. Here, we use notations in Definition 3.19, Set

$$
P_{\sigma}=P\left(f_{1}, f_{2}, \ldots, f_{l}\right)_{\left(0,0, \ldots, 0, z_{0}\right)}
$$

for $\left.\left(0,0, \ldots, 0, z_{0}\right) \in \mathbb{T}_{N}(\sigma, \mathcal{L})\right|_{U}$. Let $\tilde{\Sigma}$ be a smooth complete fan which is a subdivision of $\Sigma, \tilde{\sigma} \in \tilde{\Sigma}_{\max }$ be a cone such that $\tilde{\sigma} \subset \sigma, \tilde{v}_{1}, \tilde{v}_{2}, \ldots, \tilde{v}_{n}$ be $N$-minimal generators of $\tilde{\sigma}$, and $\left(\tilde{x}_{1}, \tilde{x}_{2}, \ldots, \tilde{x}_{n}, z\right)$ be the canonical coordinates system of $\left.\mathbb{T}_{N}(\tilde{\sigma}, \mathcal{L})\right|_{U}$ associated to $\tilde{v}_{1}, \tilde{v}_{2}, \ldots, \tilde{v}_{n}$. For the point $\left(0,0, \ldots, 0, z_{0}\right)$, let us set

$$
P_{\tilde{\sigma}}=P\left(\mu^{*} f_{1}, \mu^{*} f_{2}, \ldots, \mu^{*} f_{l}\right)_{\left(0,0, \ldots, 0, z_{0}\right)},
$$

and assume that $f_{\nu}$ is expanded as

$$
f_{\nu}\left(\tilde{x}_{1}, \tilde{x}_{2}, \ldots, \tilde{x}_{n}, z\right)=\sum_{\left(a_{j}\right)_{j} \geq 0} \prod_{j=1}^{n}\left(x_{j}\right)^{a_{j}} A_{\nu,\left(a_{j}\right)_{j}}(z)
$$

around $\left(0,0, \ldots, 0, z_{0}\right)$. Then, by Lemma 3.11, $\mu^{*} f_{\nu}$ can be written as

$$
\mu^{*} f_{\nu}\left(\tilde{x}_{1}, \tilde{x}_{2}, \ldots, \tilde{x}_{n}, z\right)=\sum_{\left(a_{j}\right)_{j} \geq 0} \prod_{k=1}^{n}\left(\tilde{x}_{k}\right)^{\sum_{j=1}^{n} a_{j}\left\langle v^{j}, \tilde{v}_{k}\right\rangle} A_{\nu,\left(a_{j}\right)_{j}}(z)
$$

around $\left(0,0, \ldots, 0, z_{0}\right)$. Thus, it follows that the following two sets are same;

$$
\bigcup_{\nu=1}^{l}\left\{\sum_{j=1}^{n} a_{j} \cdot v^{j} \mid A_{\nu,\left(a_{j}\right)_{j}} \not \equiv 0\right\}=\bigcup_{\nu=1}^{l}\left\{\sum_{j, k=1}^{n} a_{j}\left\langle v^{j}, \tilde{v}_{k}\right\rangle \cdot \tilde{v}^{k} \mid A_{\nu,\left(a_{j}\right)_{j}} \not \equiv 0\right\} .
$$


However, since the two signature $\overline{ } \bar{*}$ appeared in the definition of $P_{\sigma}$ and $P_{\tilde{\sigma}}$ are different from each other, we can not say nothing more than $P_{\sigma} \subset P_{\tilde{\sigma}}$ in general.

REMARK 3.21. Here, we use notations in Definition 3.19. We remark that $P\left(f_{1}, f_{2}, \ldots, f_{l}\right)_{\left(\left(x_{0}\right)_{1},\left(x_{0}\right)_{2}, \ldots,\left(x_{0}\right)_{n}, z_{0}\right)}$ is finitely generated in the following sense; There exists a finite subset

$$
\left\{m_{1}, m_{2}, \ldots, m_{l}\right\} \subset P\left(f_{1}, f_{2}, \ldots, f_{l}\right)_{\left(\left(x_{0}\right)_{1},\left(x_{0}\right)_{2}, \ldots,\left(x_{0}\right)_{n}, z_{0}\right)} \cap \bigoplus_{j=1}^{n} \mathbb{Z}_{\geq 0} v^{j}
$$

of the lattice such that the equation

$$
P\left(f_{1}, f_{2}, \ldots, f_{l}\right)_{\left(\left(x_{0}\right)_{1},\left(x_{0}\right)_{2}, \ldots,\left(x_{0}\right)_{n}, z_{0}\right)}=\overline{\overline{\left\{m_{1}, m_{2}, \ldots, m_{l}\right\}}}
$$

holds. More generally, for any subset $A \subset \bigoplus_{j=1}^{n} \mathbb{Z}_{\geq 0} v^{j}$, there exists a finite subset

$$
\left\{m_{1}, m_{2}, \ldots, m_{l}\right\} \subset \overline{\bar{A}} \cap \bigoplus_{j=1}^{n} \mathbb{Z}_{\geq 0} v^{j}
$$

of lattice points such that the equation $\overline{\bar{A}}=\overline{\overline{\left\{m_{1}, m_{2}, \ldots, m_{l}\right\}}}$ holds.

LEMmA 3.22. For each finite set $A \subset \bigoplus_{j=1}^{n} \mathbb{Q}_{\geq 0} v^{j}$ of rational points, there exists a smooth complete cone $\tilde{\Sigma}$ which satisfies the following two conditions (i) and (ii). (i) $\tilde{\Sigma}$ is a subdivision of $\Sigma$. (ii) For all n-dimensional cone $\tilde{\sigma} \in \tilde{\Sigma}$ satisfying $\tilde{\sigma} \subset \sigma$, there exists an element $m_{0} \in A$ such that $\min _{m \in \overline{\bar{A}}}\langle m, w\rangle=\left\langle m_{0}, w\right\rangle$ holds for all $w \in \tilde{\sigma}$, where $\tilde{v}_{1}, \tilde{v}_{2}, \ldots, \tilde{v}_{n}$ is $N$-minimal generators of $\tilde{\sigma}$.

Proof. Let $\tilde{\Sigma}$ be a fan which is made by cutting all cones of $\Sigma$ by the all hyperplanes

$$
\left\{w \in N_{\mathbb{R}} \mid\left\langle m_{j}, w\right\rangle=\left\langle m_{k}, w\right\rangle\right\}\left(m_{j}, m_{k} \in A\right)
$$

of $N_{\mathbb{R}}$. Since $A \subset \bigoplus_{j=1}^{n} \mathbb{Q}_{\geq 0} v^{j}$, each cone of $\tilde{\Sigma}$ is rational. Moreover, for all $n$-dimensional cone of $\tilde{\Sigma}$ satisfying $\tilde{\sigma} \subset \sigma$, there exists an element $m_{\tilde{\sigma}} \in A$ such that $\min _{m \in \overline{\bar{A}}}\langle m, w\rangle=$ $\left\langle m_{\tilde{\sigma}}, w\right\rangle$ holds for all $w \in \tilde{\sigma}$. Let $\tilde{\Sigma}^{\prime}$ be a smooth fan which is a subdivision of $\tilde{\Sigma}$. This fan $\tilde{\Sigma}^{\prime}$ is what we desired.

\section{Construction of minimal Singular metrics}

Here, we use notations in the previous section. In this section, we construct a minimal singular metric on the big line bundle $L=\pi^{*} L_{0} \otimes \mathcal{O}_{X}\left(D_{h}\right)$ over the total space of a toric bundle $X=\mathbb{T}_{N}(\Sigma, \mathcal{L})$ over a complex torus $V$, where $\Sigma$ is a smooth projective fan in a $n$-dimensional fan $N$. According to Proposition 3.4, it is clear that the set $\square_{\mathrm{Nef}}\left(L_{0}, h\right)=\square_{\mathrm{Nef}}\left(L_{0}, h\right)$ is not empty in this setting.

First of all, we define the singular hermitian metric $e^{-\psi_{\sigma, m}}$ for each $m \in \square_{\mathrm{Nef}}\left(L_{0}, h\right)$. 
Definition 4.1. Let $m$ be an element of $\square_{\text {Nef }}\left(L_{0}, h\right), \sigma$ be an element of $\Sigma_{\max }, v_{1}, v_{2}$, $\ldots, v_{n}$ be $N$-minimal generators of $\sigma$, and $\left\{m_{\sigma}\right\}_{\sigma}$ be the Cartier data of $D_{h}$. Here, we define the plurisubharmonic function $\psi_{\sigma, m}$ on $\left.\mathbb{T}_{N}(\sigma, \mathcal{L})\right|_{U}$ by

$$
\psi_{\sigma, m}\left(x_{1}, x_{2}, \ldots, x_{n}, z\right)=\log \left(\prod_{j=1}^{n}\left|x_{j}\right|^{2\left\langle m-m_{\sigma}, v_{j}\right\rangle}\right)+\varphi_{L_{0} \otimes \mathcal{L}^{m}}(z),
$$

where $U$ is a sufficiently small open set in $V$ and $\varphi_{L_{0} \otimes \mathcal{L}^{m}}=\varphi_{L_{0}}+\sum_{j=1}^{n}\left\langle m, v_{j}\right\rangle \varphi_{\mathcal{L}^{v_{j}}}$. For the definition of $\varphi_{L_{0}}$ and $\varphi_{\mathcal{L}^{v} j}$, see Proposition 2.3. And here, we formally regard $0^{0}$ as 1 .

REMARK 4.2. In Definition 4.1, the first term of the defining equation of $\psi_{\sigma, m}$ is clearly plurisubharmonic. According to Proposition 2.3, the second term is also turned out to be plurisubharmonic. Thus $\psi_{\sigma, m}$ is also a plurisubharmonic function, indeed.

REMARK 4.3. The functions $\left\{e^{-\psi_{\sigma, m}}\right\}_{\sigma \in \Sigma_{\max }}$ glue together to give a singular hermitian metric on $L$. Here, we explain this fact when $m$ is a rational point of $M_{\mathbb{R}}$ for simplicity.

Let $\nu$ be a natural number such that $\nu m \in M$. By Observation 3.5, $\nu \psi_{\sigma, m}$ can be rewritten as

$$
\nu \psi_{\sigma, m}=\log \left|\chi^{\nu m}\right|^{2}+\nu \varphi_{L_{0} \otimes \mathcal{L}^{m}}
$$

Since $\chi^{\nu m}$ can be regarded as a meromorphic section of the line bundle $\mathcal{O}_{X}\left(D_{\nu h}\right) \otimes \pi^{*} \mathcal{L}^{-\nu m}$, the first term of the right hand side of the above equation is turned out to be a local weight of a singular hermitian metric which is defined globally on $\mathcal{O}_{X}\left(D_{\nu h}\right) \otimes \pi^{*} \mathcal{L}^{-\nu m}$. Since the second term is also a local weight of the hermitian metric globally defined on $\pi^{*}\left(L_{0}^{\nu} \otimes \mathcal{L}^{\nu m}\right)$, the sum $\nu \psi_{\sigma, m}$ is a local weight of a singular hermitian metric globally defined on $\nu L=\pi^{*} L_{0}^{\nu} \otimes \mathcal{O}_{X}\left(D_{\nu h}\right)$.

This explanation also makes sense in the general case, by considering formally with $\mathbb{R}$-line bundles.

Definition 4.4. We define the plurisubharmonic function $\psi_{\sigma}$ on $\left.\mathbb{T}_{N}(\sigma, \mathcal{L})\right|_{U}$ by

$$
\psi_{\sigma}\left(x_{1}, x_{2}, \ldots, x_{n}, z\right)=\max _{m \in \square_{\text {Nef }}\left(L_{0}, h\right)} \psi_{\sigma, m}\left(x_{1}, x_{2}, \ldots, x_{n}, z\right)
$$

for a sufficiently small open set $U$ of $V$ and $\sigma \in \Sigma_{\max }$.

REMARK 4.5. Since each $\psi_{\sigma, m}$ is plurisubharmonic, it is clear that the upper envelope

$$
\left(x_{1}, x_{2}, \ldots, x_{n}, z\right) \longmapsto \limsup _{\left(\xi^{1}, \xi^{2}, \ldots, \xi^{n}, \zeta\right) \rightarrow\left(x_{1}, x_{2}, \ldots, x_{n}, z\right)} \psi_{\sigma}\left(\xi^{1}, \xi^{2}, \ldots, \xi^{n}, \zeta\right)
$$

of $\psi_{\sigma}$ is a plurisubharmonic function. Now let us consider the function

$$
\left(\left(x_{1}, x_{2}, \ldots, x_{n}, z\right), m\right) \longmapsto e^{\psi_{\sigma, m}\left(x_{1}, x_{2}, \ldots, x_{n}, z\right)}=\left(\prod_{j=1}^{n}\left|x_{j}\right|^{2\left\langle m-m_{\sigma}, v_{j}\right\rangle}\right) \cdot e^{\varphi_{L_{0} \otimes \mathcal{L}^{m}}(z)} .
$$


This function is a continuous function defined on $\left.\mathbb{T}_{N}(\sigma, \mathcal{L})\right|_{U} \times \square_{\text {Nef }}\left(L_{0}, h\right)$. Since $\square_{\text {Nef }}\left(L_{0}, h\right)$ is compact (Lemma 3.2), the function

$$
\left(\left(x_{1}, x_{2}, \ldots, x_{n}, z\right), m\right) \longmapsto e^{\psi_{\sigma}\left(x_{1}, x_{2}, \ldots, x_{n}, z\right)}=\max _{m \in \square_{\mathrm{Nef}}\left(L_{0}, h\right)} e^{\psi_{\sigma, m}\left(x_{1}, x_{2}, \ldots, x_{n}, z\right)},
$$

is also continuous. Therefore, $\psi_{\sigma}$ itself is also a plurisubharmonic function.

REMARK 4.6. Remark 4.3 yields that $\left\{e^{-\psi_{\sigma}}\right\}_{\sigma \in \Sigma_{\max }}$ glue together to give a singular hermitian metric on $L$ whose curvature current is semi-positive.

TheOREM 4.7. Assume that $L$ is a big line bundle, then the singular hermitian metric $e^{-\psi_{\sigma}}$ of $L$ is a minimal singular metric.

From now on, we will prepare for the proof of Theorem 4.7. Let $\sigma \in \Sigma$ be a $n$ dimensional cone, $v_{1}, v_{2}, \ldots, v_{n}$ be $N$-minimal generators of $\sigma$, and $\left(x_{1}, x_{2}, \ldots, x_{n}, z\right)$ be the canonical coordinates system of $\left.\mathbb{T}_{N}(\sigma, \mathcal{L})\right|_{U}$ associated to $v_{1}, v_{2}, \ldots, v_{n}$, where $U$ is a sufficiently small open set in $V$. We use these notations throughout this section.

LEMmA 4.8. Let us fix a point $\left.\left(\left(x_{0}\right)_{1},\left(x_{0}\right)_{2}, \ldots,\left(x_{0}\right)_{n}, z_{0}\right) \in \mathbb{T}_{N}(\sigma, \mathcal{L})\right|_{U}$, and denote by $I$ the set $\left\{j \in\{1,2, \ldots, n\} \mid x_{0}^{j}=0\right\}$. Then, there exist constants $C_{1}$ and $C_{2}$ such that

$$
\max _{m \in \square_{\mathrm{Nef}}\left(L_{0}, h\right)} \log \prod_{j \in I}\left|x_{j}\right|^{2\left\langle m-m_{\sigma}, v_{j}\right\rangle}+C_{1} \leq \psi_{\sigma} \leq \max _{m \in \square_{\mathrm{Nef}}\left(L_{0}, h\right)} \log \prod_{j \in I}\left|x_{j}\right|^{2\left\langle m-m_{\sigma}, v_{j}\right\rangle}+C_{2}
$$

holds on $\prod_{j \in I}^{n}\left\{\left|x_{j}\right| \leq 1\right\} \times \prod_{j \notin I}\left\{\left|x_{j}-x_{0}^{j}\right| \leq \delta_{j}\right\} \times \bar{U}$, where $\left\{\delta_{j}\right\}_{j \notin I}$ is a system of sufficiently small positive numbers such that $0 \notin\left\{\left|x_{j}-x_{0}^{j}\right| \leq \delta_{j}\right\}$ for all $j \notin I$, and $m_{\sigma}$ is the element of the Cartier data of $D_{h}$ which is associated to $\sigma$.

Proof. The function

$$
\left(m,\left(x_{j}\right)_{j \notin I}, z\right) \longmapsto \log \prod_{j \notin I}\left|x_{j}\right|^{2\left\langle m-m_{\sigma}, v_{j}\right\rangle}+\varphi_{L_{0} \otimes \mathcal{L}^{m}}(z)
$$

defined on $\square_{\text {Nef }}\left(L_{0}, h\right) \times \prod_{j \notin I}\left\{\left|x_{j}-x_{0}^{j}\right| \leq \delta_{j}\right\} \times \bar{U}$ is continuous. According to Lemma 3.2, $\square_{\mathrm{Nef}}\left(L_{0}, h\right) \times \prod_{j \notin I}\left\{\left|x_{j}-x_{0}^{j}\right| \leq \delta_{j}\right\} \times \bar{U}$ is compact, which yields that this function has both the maximum value and the minimum value, which we denote by $C_{1}$ and $C_{2}$ respectively. Therefore, the inequality

$$
\log \prod_{j \in I}\left|x_{j}\right|^{2\left\langle m-m_{\sigma}, v_{j}\right\rangle}+C_{1} \leq \psi_{\sigma, m} \leq \log \prod_{j \in I}\left|x_{j}\right|^{2\left\langle m-m_{\sigma}, v_{j}\right\rangle}+C_{2}
$$

follows, which proves the lemma.

As we have assumed that $L$ is big thus in particular pseudo-effective, there must be a minimal singular metric on $L$. We fix one of these and denote it by $h_{\min }$. 
LEMMA 4.9. Let $\sigma$ be an element of $\Sigma_{\max }$, and we denote the weight function of $h_{\min }$ around $\left.\mathbb{T}_{N}(\sigma, \mathcal{L})\right|_{\bar{U}}$ with respect to the local trivialization of $L$ as in Observation 3.5 by $\varphi_{\min , \sigma}$. Then, there exists a constant $C_{\sigma}$ such that

$$
\varphi_{\min , \sigma} \leq \psi_{\sigma}+C_{\sigma}
$$

holds on the set $K_{\sigma}=\left\{\left.\left(x_{1}, x_{2}, \ldots, x_{n}, z\right) \in \mathbb{T}_{N}(\sigma, \mathcal{L})\right|_{\bar{U}}|\forall j \in\{1,2, \ldots, n\},| x_{j} \mid \leq 1\right\}$.

Proof. Let us denote by $m_{\sigma}$ the element of the Cartier data of $D_{h}$ associated to $\sigma$. Applying Lemma 4.8 with $I=\{1,2, \ldots, n\}$, it follows that there exists a constant $C$ such that

$$
\max _{m \in \square_{\text {Nef }}\left(L_{0}, h\right)} \log \prod_{j=1}^{n}\left|x_{j}\right|^{2\left\langle m-m_{\sigma}, v_{j}\right\rangle} \leq \psi_{\sigma}+C
$$

holds on $K_{\sigma}$.

Thus here, we compare $\varphi_{\min , \sigma}$ with $\max _{m \in \square_{\mathrm{Nef}}\left(L_{0}, h\right)} \log \prod_{j=1}^{n}\left|x_{j}\right|^{2\left\langle m-m_{\sigma}, v_{j}\right\rangle}$.

We choose an infinite subsequence $\{\nu\} \subset \mathbb{N}$ and a finite subset $\left\{f_{j}^{(\nu)}\right\}_{1 \leq j \leq N_{\nu}}$ of $H^{0}(X, \nu L)$ for each $\nu$ satisfying the following condition; The function

$$
\varphi_{\nu}=\frac{1}{\nu} \log \sum_{j=1}^{N_{\nu}}\left|f_{j}^{(\nu)}\right|^{2}
$$

converges pointwise to $\varphi_{\min , \sigma}$ on $X$ except a subset of measure 0 as $\nu \rightarrow \infty$, and the maximum value $M_{\varphi_{\nu}}$ of $\varphi_{\nu}$ on $K_{\sigma}$ also converges to $M_{\varphi_{\min }, \sigma}=\max _{K_{\sigma}} \varphi_{\min , \sigma}$ as $\nu \rightarrow \infty$. The existence of these functions can be immediately shown by applying [6, Theorem (13.21)] regarding $\varphi$ in the theorem as $\left(1-\frac{1}{k}\right) \varphi_{\min }+\frac{1}{k} \varphi_{+}$for each natural number $k$, where $\varphi_{+}$is the local weight of a singular hermitian metric $h_{+}$on $L$ which satisfies $\Theta_{h_{+}} \geq \varepsilon \omega$ for some positive number $\varepsilon$ and a Kähler metric $\omega$ on $X$.

Then, according to the next Lemma 4.10, an inequality

$$
\varphi_{\nu} \leq \max _{m \in \square_{\mathrm{Nef}}\left(L_{0}, h\right)} \log \prod_{j=1}^{n}\left|x_{j}\right|^{2\left\langle m-m_{\sigma}, v_{j}\right\rangle}+M_{\varphi_{\nu}}
$$

holds on $K_{\sigma}$. Considering this inequality as $\nu \rightarrow \infty$, we obtain

$$
\varphi_{\min , \sigma} \leq \max _{m \in \square_{\mathrm{Nef}}\left(L_{0}, h\right)} \log \prod_{j=1}^{n}\left|x_{j}\right|^{2\left\langle m-m_{\sigma}, v_{j}\right\rangle}+M_{\varphi_{\min }, \sigma}
$$

on $K_{\sigma}$ except the subset of measure 0 . Since the both hand sides are plurisubharmonic, this inequality holds on whole $K_{\sigma}$.

According to the above argument, we obtain the inequality

$$
\varphi_{\min , \sigma} \leq \psi_{\sigma}+C+M_{\varphi_{\min }, \sigma}
$$

on $K_{\sigma}$, which proves the lemma. 
Lemma 4.10. Here we use notations in the proof of Lemma 4.9. The inequality

$$
\varphi_{\nu} \leq \max _{m \in \square_{\mathrm{Nef}}\left(L_{0}, h\right)} \log \prod_{j=1}^{n}\left|x_{j}\right|^{2\left\langle m-m_{\sigma}, v_{j}\right\rangle}+M_{\varphi_{\nu}}
$$

holds on $K_{\sigma}$.

Proof. Let $P\left(\varphi_{\nu}\right)_{\sigma}:=\frac{1}{\nu} P\left(f_{1}^{(\nu)}, f_{2}^{(\nu)}, \ldots, f_{N_{\nu}}^{(\nu)}\right)_{\left(0,0, \ldots, 0, z_{0}\right)}$. According to Proposition 3.4 and Observation 3.9, $\nu P\left(\varphi_{\nu}\right)_{\sigma}$ is a subset of $S\left(L_{0}^{\nu}, \nu h\right)_{\sigma}$. Since $\square_{\mathrm{Nef}}\left(L_{0}^{\nu}, \nu h\right)=\nu \square_{\mathrm{Nef}}\left(L_{0}, h\right)$ holds, it turns out that $S\left(L_{0}^{\nu}, \nu h\right)_{\sigma}=\nu S\left(L_{0}, h\right)_{\sigma}$, thus we obtain

$$
P\left(\varphi_{\nu}\right)_{\sigma} \subset S\left(L_{0}, h\right)_{\sigma} .
$$

Therefore, according to Remark 3.18, it is sufficient to show the inequality

$$
\varphi_{\nu} \leq \max _{m \in P\left(\varphi_{\nu}\right)_{\sigma}} \log \prod_{j=1}^{n}\left|x_{j}\right|^{2\left\langle m, v_{j}\right\rangle}+M_{\varphi_{\nu}}
$$

on $K_{\sigma}$.

According to Remark 3.21, there exists a finite subset $A$ of $P\left(\varphi_{\nu}\right)$ whose elements are rational and which satisfies $P\left(\varphi_{\nu}\right)=\overline{\bar{A}}$. For this set $A$, we fix such a subdivision $\tilde{\Sigma}$ of $\Sigma$ as in Lemma 3.22. In the following, we use notations we used in Section 4.2. According to Corollary 3.15, it is sufficient to show that

$$
\mu^{*} \varphi_{\nu} \leq \mu^{*}\left(\max _{m \in P\left(\varphi_{\nu}\right)_{\sigma}} \log \prod_{j=1}^{n}\left|x_{j}\right|^{2\left\langle m, v_{j}\right\rangle}\right)+M_{\varphi_{\nu}}
$$

on $K_{\tilde{\sigma}}=\left\{\left.\left(\tilde{x}_{1}, \tilde{x}_{2}, \ldots, \tilde{x}_{n}, z\right) \in \mathbb{T}_{N}(\tilde{\sigma}, \mathcal{L})\right|_{\bar{U}}|\forall j \in\{1,2, \ldots, n\},| \tilde{x}_{j} \mid \leq 1\right\}$ for each $\tilde{\sigma} \in$ $\left(\tilde{\Sigma}_{\sigma}\right)_{\max }$.

Since

$$
\log \prod_{j=1}^{n}\left|\mu^{*} x_{j}\right|^{\left\langle\left\langle m, v_{j}\right\rangle\right.}=\log \prod_{j=1}^{n} \prod_{k=1}^{n}\left|\tilde{x}_{k}\right|^{2\left\langle m, v_{j}\right\rangle\left\langle v^{j}, \tilde{v}_{k}\right\rangle}=\sum_{k=1}^{n}\left\langle m, \tilde{v}_{k}\right\rangle \log \left|\tilde{x}_{k}\right|^{2}
$$

holds, we obtain

$$
\mu^{*}\left(\max _{m \in P\left(\varphi_{\nu}\right)_{\sigma}} \log \prod_{j=1}^{n}\left|x_{j}\right|^{2\left\langle m, v_{j}\right\rangle}\right)=\max _{m \in P\left(\varphi_{\nu}\right)_{\sigma}} \sum_{j=1}^{n}\left\langle m, \tilde{v}_{j}\right\rangle \log \left|\tilde{x}_{j}\right|^{2} .
$$

As $\log \left|\tilde{x}_{j}\right|^{2} \leq 0$ holds for all $j$ on $K_{\tilde{\sigma}}$, the equation we desire can be rewritten as

$$
\mu^{*} \varphi_{\nu} \leq \log \prod_{j=1}^{n}\left|\tilde{x}_{j}\right|^{2\left\langle m_{0}, \tilde{v}_{j}\right\rangle}+M_{\varphi_{\nu}}
$$

where $m_{0} \in P\left(\varphi_{\nu}\right)_{\sigma}$ is such an element as in Lemma 3.22.

Let $P\left(\varphi_{\nu}\right)_{\tilde{\sigma}}:=\frac{1}{\nu} P\left(\mu^{*} f_{1}^{(\nu)}, \mu^{*} f_{2}^{(\nu)}, \ldots, \mu^{*} f_{N_{\nu}}^{(\nu)}\right)_{\left(0,0, \ldots, 0, z_{0}\right)}$. According to Remark 3.20, and since both $P\left(\varphi_{\nu}\right)_{\tilde{\sigma}}$ and $P\left(\varphi_{\nu}\right)_{\sigma}$ are generated by the same set, it turns out that $\mu^{*} f_{j}^{(\nu)}$ 
can be divided by the function $\prod_{k=1}^{n}\left(x_{k}\right)^{\left\langle\nu m_{0}, \tilde{v}_{k}\right\rangle}$ for all $j \in\left\{1,2, \ldots, N_{\nu}\right\}$. Denoting the quotient by $g_{j}^{(\nu)}$, the function $\mu^{*} \varphi_{\nu}-\log \prod_{j \in I}\left|\tilde{x}_{j}\right|^{2\left\langle m_{0}, \tilde{v}_{j}\right\rangle}$ can be rewritten as

$$
\mu^{*} \varphi_{\nu}-\log \prod_{j=1}^{n}\left|\tilde{x}_{j}\right|^{2\left\langle m_{0}, \tilde{v}_{j}\right\rangle}=\frac{1}{\nu} \log \sum_{j=1}^{N_{\nu}}\left|g_{j}^{(\nu)}\right|^{2} .
$$

Thus, this function is a plurisubharmonic function on $K_{\tilde{\sigma}}$, and it has the maximum value on $K_{\tilde{\sigma}}$, which we denote by $M_{\varphi_{\nu}, \tilde{\sigma}}$. Then, since

$$
\mu^{*} \varphi_{\nu} \leq \log \prod_{j=1}^{n}\left|\tilde{x}_{j}\right|^{2\left\langle m_{0}, \tilde{v}_{j}\right\rangle}+M_{\varphi_{\nu}, \tilde{\sigma}}
$$

holds on $K_{\tilde{\sigma}}$. Therefore, it remains to prove that $M_{\varphi_{\nu}, \tilde{\sigma}} \leq M_{\varphi_{\nu}}$.

Assume that the plurisubharmonic function $\mu^{*} \varphi_{\nu}-\log \prod_{j \in I}\left|\tilde{x}_{j}\right|^{2\left\langle m_{0}, \tilde{v}_{j}\right\rangle}$ has the maximum value at the point $\left(\left(\tilde{x}_{0}\right)_{1},\left(\tilde{x}_{0}\right)_{2}, \ldots,\left(\tilde{x}_{0}\right)_{n}, z_{0}\right) \in K_{\tilde{\sigma}}$. We may assume $\left|\left(\tilde{x}_{0}\right)_{j}\right|=1$ for all $j$ after we change the point $\left(\left(\tilde{x}_{0}\right)_{1},\left(\tilde{x}_{0}\right)_{2}, \ldots,\left(\tilde{x}_{0}\right)_{n}, z_{0}\right) \in K_{\tilde{\sigma}}$ if necessary. It is because, in the case when $\left|\left(\tilde{x}_{0}\right)_{1}\right|<1$ for example, by considering the plurisubharmonic function

$$
\tilde{x}_{1} \mapsto \mu^{*} \varphi_{\nu}\left(\tilde{x}_{1},\left(\tilde{x}_{0}\right)_{2},\left(\tilde{x}_{0}\right)_{3}, \ldots,\left(\tilde{x}_{0}\right)_{n}, z_{0}\right)-\log \left(\left|\tilde{x}_{1}\right|^{2\left\langle m_{0}, \tilde{v}_{1}\right\rangle} \cdot \prod_{j=2}^{n}\left|\left(\tilde{x}_{0}\right)_{j}\right|^{2\left\langle m_{0}, \tilde{v}_{j}\right\rangle}\right)
$$

defined on $\left\{\left|\tilde{x}_{1}\right|<1\right\}$, the value of the function above must constantly be $M_{\varphi_{\nu}, \tilde{\sigma}}$.

Then, we can calculate that

$$
M_{\varphi_{\nu}, \tilde{\sigma}}=\mu^{*} \varphi_{\nu}\left(\left(\tilde{x}_{0}\right)_{1},\left(\tilde{x}_{0}\right)_{2}, \ldots,\left(\tilde{x}_{0}\right)_{n}, z_{0}\right)-\log \prod_{j=1}^{n}\left|\left(\tilde{x}_{0}\right)_{j}\right|^{2\left\langle m_{0}, \tilde{v}_{j}\right\rangle}=\varphi_{\nu}\left(\mu\left(\left(\tilde{x}_{0}\right)_{1},\left(\tilde{x}_{0}\right)_{2}, \ldots,\left(\tilde{x}_{0}\right)_{n}, z_{0}\right)\right) .
$$

Since $\mu\left(\left(\tilde{x}_{0}\right)_{1},\left(\tilde{x}_{0}\right)_{2}, \ldots,\left(\tilde{x}_{0}\right)_{n}, z_{0}\right) \in K_{\sigma}$, the value is at most $M_{\varphi_{\nu}}$.

Proof of Proposition 4.7. Let us denote by $h$ the singular hermitian metric defined by $\left\{e^{-\psi_{\sigma}}\right\}_{\sigma}$, and by $h_{\infty}$ a smooth hermitian metric on $L$. Then, there exist upper semicontinuous functions $\varphi_{\min }^{\prime}$ and $\psi^{\prime}$ on $X$ such that

$$
h_{\min }=h_{\infty} e^{-\varphi_{\min }^{\prime}}, h=h_{\infty} e^{-\psi^{\prime}}
$$

hold. Here, it is sufficient to prove that there exists a constant $C$ such that

$$
\varphi_{\min }^{\prime} \leq \psi^{\prime}+C
$$

holds on $\pi^{-1}(\bar{U}) \subset X$.

According to Lemma 4.9, for each $\sigma \in \Sigma_{\max }$, there exists a constant $C_{\sigma}$ such that

$$
\varphi_{\min }^{\prime} \leq \psi^{\prime}+C_{\sigma}
$$

holds on the set $K_{\sigma}=\left\{\left.\left(x_{1}, x_{2}, \ldots, x_{n}, z\right) \in \mathbb{T}_{N}(\Sigma, \mathcal{L})\right|_{\bar{U}}|\forall j \in\{1,2, \ldots, n\},| x_{j} \mid \leq 1\right\}$. Thus, according to Lemma 3.10 .

$$
\varphi_{\min }^{\prime} \leq \psi^{\prime}+C
$$

holds on $\pi^{-1}(\bar{U}) \subset X$, where $C=\max _{\sigma \in \Sigma_{\max }} C_{\sigma}$. 


\section{Properties Related to the Singularities of Minimal Singular metrics}

\subsection{Kiselman numbers and Lelong numbers of minimal singular metrics and}

Non-nef loci. Let $X$ be a smooth projective variety and $L$ be a holomorphic line bundle over $X$. According to [3, 3.6], the next proposition follows.

Proposition 5.1. If $L$ is big, then the non-nef locus $\operatorname{NNef}(L)$ of $L$ can be written as

$$
\operatorname{NNef}(L)=\left\{x \in X \mid \nu\left(\varphi_{\min }, x\right)>0\right\}
$$

where $e^{-\varphi_{\min }}$ is a minimal singular metric on $L$.

According to this proposition, we can specify the non-nef locus of a big line bundle by calculating the Lelong number of a minimal singular metric. It can be done, actually, in our setting.

Proposition 5.2. Let $X$ be the total space of a toric bundle $\mathbb{T}_{N}(\Sigma, \mathcal{L})$ over a complex torus and $L=\pi^{*} L_{0} \otimes \mathcal{O}_{X}\left(D_{h}\right)$ be a big line bundle over $X$, where $\Sigma i$ s a smooth projective fan in a $n$-dimensional lattice $N$. The Kiselman number

$$
\nu_{\zeta, w}^{K}\left(\varphi_{\min }, x_{0}\right)=\sup \left\{t \geq\left. 0\left|\varphi_{\min } \leq t \log \sum_{j=1}^{n+d}\right| \zeta_{j}\right|^{2 w_{j}}+O(1) \text { around } x_{0}\right\}
$$

associated to the coordinates system

$$
\zeta=\left(\zeta_{1}, \zeta_{2}, \ldots, \zeta_{n+d}\right)=\left(x_{1}, x_{2}, \ldots, x_{n}, z_{1}, z_{2}, \ldots, z_{d}\right)
$$

and $w=\left(w_{j}\right) \in \bigoplus_{j \in I} \mathbb{R}_{>0}$ of a minimal singular metric $e^{-\varphi_{\min }}$ at a point $x_{0}=\left(\left(x_{0}\right)_{1},\left(x_{0}\right)_{2}, \ldots,\left(x_{0}\right)_{n}, z_{0}\right) \in \mathbb{T}_{N}(\sigma, \mathcal{L})$ (see [1, Section 5.2] for the definition) can be calculated by using notations in the previous section that

$$
\nu_{\zeta, w}^{K}\left(\varphi_{\min }, x_{0}\right)=\min _{m \in S\left(L_{0}, h\right)_{\sigma}}\left\langle m, \sum_{j \in I} \frac{v_{j}}{w_{j}}\right\rangle
$$

where we denote by $I$ the set $\left\{j \mid x_{0}^{j}=0\right\}$ and by $\left(x_{1}, x_{2}, \ldots, x_{n}, z_{1}, z_{2}, \ldots, z_{d}\right)$ the canonical coordinates system of $\left.\mathbb{T}_{N}(\sigma, \mathcal{L})\right|_{U}$ associated to $N$-minimal generators $v_{1}, v_{2}, \ldots, v_{n}$ of $\sigma$. Especially, the Lelong number at $x_{0}$ can be calculated that

$$
\nu\left(\varphi_{\min }, x_{0}\right)=\min _{m \in S\left(L_{0}, h\right)_{\sigma}} \sum_{j \in I}\left\langle m, v_{j}\right\rangle .
$$

Corollary 5.3. Let $X, L$ be as that of the previous proposition. The following conditions are equivalent.

(1) $\varphi_{\min }\left(x_{0}\right)\left(=\psi_{\sigma}\left(x_{0}\right)\right)=-\infty$.

(2) $\psi_{\sigma}$ is not continuous at $x_{0}$.

(3) $\nu\left(\varphi_{\min }, x_{0}\right)\left(=\nu\left(\psi_{\sigma}, x_{0}\right)\right)>0$. 
especially,

$$
\varphi_{\min }^{-1}(-\infty)=\operatorname{Pole}\left(\varphi_{\min }\right)
$$

holds, where we denote by $\operatorname{Pole}\left(\varphi_{\min }\right)$ the set $\left\{x \in X \mid \nu\left(\varphi_{\min }, x\right)>0\right\}$.

The next proposition is also obtained easily by Theorem 4.7 .

Proposition 5.4. Let $X, L$ be as that of Proposition 5.2. Then, Pole $\left(\varphi_{\min }\right)$ is a Zariski closed set.

According to these argument, we obtain the following corollary.

Corollary 5.5. Let $X$ be the total space of a toric bundle $\mathbb{T}_{N}(\Sigma, \mathcal{L})$ over a complex torus and $L=\pi^{*} L_{0} \otimes \mathcal{O}_{X}\left(D_{h}\right)$ be a big line bundle over $X$, where $\Sigma$ is a smooth projective fan. Then, the set $\operatorname{NNef}(L)$ is a Zariski closed subset of $X$.

5.2. Multiplier ideal sheaves. Let $\Sigma$ be a smooth projective fan of a $n$-dimensional lattice $N$. Fix $N$-minimal generators $v_{1}, v_{2}, \ldots, v_{n}$ of $\sigma \in \Sigma_{\max }$. Let $\left(x_{1}, x_{2}, \ldots, x_{n}, z\right)$ be the canonical coordinates system of $\left.\mathbb{T}_{N}(\sigma, \mathcal{L})\right|_{U}$ associated to $v_{1}, v_{2}, \ldots, v_{n}$, where $U$ is a sufficiently small open set in $V$. In this section, we consider the condition

$$
f \in \mathcal{J}\left(h_{\min }^{t}\right)_{\left(\left(x_{0}\right)_{1},\left(x_{0}\right)_{2}, \ldots,\left(x_{0}\right)_{n}, z_{0}\right)},
$$

where $\left(\left(x_{0}\right)_{1},\left(x_{0}\right)_{2}, \ldots,\left(x_{0}\right)_{n}, z_{0}\right)$ is a point of $\left.\mathbb{T}_{N}(\sigma, \mathcal{L})\right|_{U}, f$ is an element of $\mathcal{O}_{X,\left(\left(x_{0}\right)_{1},\left(x_{0}\right)_{2}, \ldots,\left(x_{0}\right)_{n}, z\right)} \backslash$ $\{0\}, t$ is a positive real number, and $h_{\min }$ is a minimal singular metric on $L$. In the following, we also denote by $\mathcal{J}\left(t \varphi_{\text {min }}\right)$ the multiplier ideal sheaf $\mathcal{J}\left(h_{\text {min }}^{t}\right)$ by using the local weight function $\varphi_{\min }$ of the singular hermitian metric $h_{\min }$.

Let $I:=\left\{j \in\{1,2, \ldots, n\} \mid\left(x_{0}\right)_{j}=0\right\}$. For this set $I$, let us denote the expansion appeared in Definition 3.19 by

$$
f\left(x_{1}, x_{2}, \ldots, x_{n}, z\right)=\sum_{m \in \operatorname{Pr}^{I}\left(\sigma^{\vee} \cap M\right)} \prod_{j \in I}\left(x_{j}\right)^{\left\langle m, v_{j}\right\rangle} A_{m}\left(x_{I^{c}}, z\right),
$$

where the map $\operatorname{Pr}^{I}$ is the projection from $M_{\mathbb{R}}$ to $\operatorname{Span}_{\mathbb{R}}\left\{v^{j}\right\}_{j \in I}$. As the dual version of this map, we denote the projection from $N_{\mathbb{R}}$ to $\operatorname{Span}_{\mathbb{R}}\left\{v_{j}\right\}_{j \in I}$ by $\operatorname{Pr}_{I}$ in the following. Fix a set $\left.A \subset P(f),\left(x_{0}\right)_{1},\left(x_{0}\right)_{2}, \ldots,\left(x_{0}\right)_{n}, z_{0}\right)$ of lattice points such that

$$
P(f)_{\left(\left(x_{0}\right)_{1},\left(x_{0}\right)_{2}, \ldots,\left(x_{0}\right)_{n}, z_{0}\right)}=\overline{\bar{A}}
$$

holds.

Corollary 5.6. The followings are equivalent.

(1) $f \in \mathcal{J}\left(t \varphi_{\min }\right)_{\left(\left(x_{0}\right)_{1},\left(x_{0}\right)_{2}, \ldots,\left(x_{0}\right)_{n}, z_{0}\right)}$.

(2) $\min _{m \in t S\left(L_{0}, h\right)_{\sigma}}\langle m, w\rangle<\left\langle m_{0}+\sum_{j \in I} v^{j}, w\right\rangle$ for all $m_{0} \in A$ and $w \in \operatorname{Pr}_{I}(\sigma) \backslash\{0\}$. 
Corollary 5.6 immediately follows from Theorem 4.7 and the result of Guenancia [10] referring to the way to compute the multiplier ideal sheaves associated to "toric plurisubharmonic functions", which can be regarded as a generalization of the famous Howald's result ([11, Theorem 11]) in algebraic setting.

According to Corollary 5.6, [5, 1.10, 1.11], and [13, 11.2.12 (ii)], we obtain next corollary.

Corollary 5.7. Let $X$ be the total space of a smooth projective toric bundle over a complex torus, $D$ a big divisor on $X$, and $e^{-\varphi_{\min }}$ be a minimal singular metric on the line bundle $\mathcal{O}_{X}(D)$.

(1) If $f \in \mathcal{J}\left(t \varphi_{\min }\right)_{x_{0}}$ at the point $x_{0}$, then $f \in \mathcal{J}\left((1+\varepsilon) t \varphi_{\text {min }}\right)_{x_{0}}$ holds for sufficiently small positive number $\varepsilon$ and any positive real number $t$. Especially, since the sheaf $\mathcal{J}\left(t \varphi_{\text {min }}\right)$ is coherent, it follows that

$$
\mathcal{J}\left(t \varphi_{\min }\right)=\mathcal{J}_{+}\left(t \varphi_{\min }\right)
$$

(2) Let $P$ be a nef big divisor on $X$, then

$$
H^{j}\left(X, \mathcal{O}_{X}\left(K_{X}+P+L\right) \otimes \mathcal{J}\left(\varphi_{\min }\right)\right)=0
$$

holds for all $j>0$.

\section{Some examples}

In this section, we will introduce three examples for $X$ and $L$ in the previous sections. We construct them as $\mathbb{P}^{2}$-bundles over abelian surfaces, by following [14, CHAPTER IV $\S 2.6]$ basically. In this section, we use notations in Example 2.7.

As a preparation, we first recall a useful lemma to see $L$ is big.

LEMMA 6.1. In the setting of Example 2.7, $L$ is big if and only if there exists a triple $(a, b, c)$ of nonnegative integers such that $L_{0}^{a} \otimes L_{1}^{b} \otimes L_{2}^{c}$ is ample line bundle over $V$.

This lemma can be easily shown by applying the result known by Cutkosky ([12, Lemma 2.3.2]) and the fact that the ample cones of complex tori coincide with these big cones.

Let $E$ be a sufficiently general smooth elliptic curve and $o$ be a point of $E$. For example, you can choose $\mathbb{C} /(\mathbb{Z}+(\pi+\sqrt{-1}) \mathbb{Z})$ for $E$. Let

$$
V=E \times E .
$$

It is known that the rank of the Neron-Severi group $\mathrm{NS}(V)$ of $V$ is three and this group is generated by the following three classes ([12, Chapter 1.5.B]).

- $f_{1}=c_{1}\left(\mathcal{O}_{V}\left(F_{1}\right)\right)$, where $F_{1}$ stands for the prime divisor $\{o\} \times E \subset V$.

- $f_{2}=c_{1}\left(\mathcal{O}_{V}\left(F_{2}\right)\right)$, where $F_{2}$ stands for the prime divisor $E \times\{o\} \subset V$.

- $\delta=c_{1}\left(\mathcal{O}_{V}(\Delta)\right)$, where $\Delta$ stands for the prime divisor $\{(x, y) \in E \times E \mid x=y\}$. 
By using these three classes, the nef cone $\operatorname{Nef}(V)$ of $V$ can be written as

$$
\operatorname{Nef}(V)=\left\{a f_{1}+b f_{2}+c \delta \mid a, b, c \in \mathbb{R}, a b+b c+c a \geq 0, a+b+c \geq 0\right\} .
$$

In order to obtain more useful expression of $\operatorname{Nef}(V)$, let us define the other basis of $\mathrm{NS}(V) \otimes \mathbb{R}$ by

$$
l_{1}=\frac{1}{6}\left(f_{1}+f_{2}-2 \delta\right), l_{2}=\frac{1}{6}\left(-\sqrt{3} f_{1}+\sqrt{3} f_{2}\right), \text { and } l_{3}=\frac{1}{6}\left(f_{1}+f_{2}+\delta\right) .
$$

By using these classes, $\operatorname{Nef}(V)$ can be written as

$$
\operatorname{Nef}(V)=\left\{a l_{1}+b l_{2}+c l_{3} \mid c^{2} \geq a^{2}+b^{2}, c \geq 0\right\} .
$$

This expression of $\operatorname{Nef}(V)$ makes it easy to judge the nef-ness of line bundles.

ExAMPLE 6.2. The first example is an example which admits a Zariski decomposition after appropriate modifications. Let us fix two positive integers $u<v$. Let $L_{0}:=$ $\mathcal{O}_{V}\left(-u F_{1}-u F_{2}-u \Delta\right), L_{1}:=\mathcal{O}_{V}\left((u+v) F_{1}+(u+v) F_{2}+(-2 u+v) \Delta\right)$, and $L_{2}:=$ $\mathcal{O}_{V}\left((-u+v) F_{1}+(-u+v) F_{2}+(2 u+v) \Delta\right)$. Then $c_{1}\left(L_{0}\right)=-6 u l_{3}, c_{1}\left(L_{1}\right)=6\left(u l_{1}+v l_{3}\right)$, and $c_{1}\left(L_{2}\right)=6\left(-u l_{1}+v l_{3}\right)$ hold. These expressions make it clear that the line bundle $L_{1} \otimes L_{2}$ is ample and, according to Lemma 6.1, that $L$ is a big line bundle in this case.

The set $\square_{\mathrm{Nef}}\left(L_{0}, h\right)$ in this setting is rational polyhedral. More precisely, $\square_{\mathrm{Nef}}\left(L_{0}, h\right)$ is the convex closure of the five points $e^{1}, e^{2}, \frac{u}{v} e^{2}, \frac{u}{2(u+v)} e^{1}+\frac{u}{2(u+v)} e^{2}, \frac{u}{v} e^{1}$ in $M_{\mathbb{R}}$. So, by applying Theorem 4.7, it immediately turns out that the weight of a minimal singular metric $\psi_{\sigma_{j}}$ satisfies $\psi_{\sigma_{j}} \sim_{\text {sing }} 1$ at any points of $X$ except for the locus $\mathbb{P}\left(L_{0}\right)$, and

$$
\begin{aligned}
\psi_{\sigma_{1}}\left(x_{1}, x_{2}, z\right) & \sim_{\text {sing }} \frac{u}{2 v(u+v)} \log \max \left\{\left|x_{1}\right|^{2(2 u+2 v)},\left|x_{2}\right|^{2(2 u+2 v)},\left|x_{1}\right|^{2 v}\left|x_{2}\right|^{2 v}\right\} \\
& \sim_{\text {sing }} \frac{u}{2 v(u+v)} \log \left(\left|x_{1}\right|^{2(2 u+2 v)}+\left|x_{2}\right|^{2(2 u+2 v)}+\left|x_{1}\right|^{2 v}\left|x_{2}\right|^{2 v}\right)
\end{aligned}
$$

at a point $\left(0,0, z_{0}\right) \in \mathbb{P}\left(L_{0}\right)$. Therefore, it follows that the non-nef locus $\operatorname{NNef}(L)$ is a Zariski closed subset $\mathbb{P}\left(L_{0}\right)$ of $X$.

According to [14, 2.5], the fact that $\square_{\mathrm{Nef}}\left(L_{0}, h\right)$ is a rational polyhedral yields that $L$ admits a Zariski decomposition after appropriate proper modifications. Especially, when $u$ and $v$ can be written as

$$
u=1, v=2 n-2
$$

for some integer $n>1,(X, L)$ is an example which admits a Zariski decomposition just after the $n$-time blow-up centered at the non-nef locus of the pull-back of $L$. It can be also checked out by using the above expression of the minimal singular metric on $L$.

According to the above expression of $\square_{\text {Nef }}\left(L_{0}, h\right)$, the result of Corollary 5.6 can be rewritten as follows. First, it is clear that $\mathcal{J}\left(h_{\text {min }}^{t}\right)$ is trivial at any point in $X \backslash \mathbb{P}\left(L_{0}\right)$. Next, for a point $x_{0} \in \mathbb{P}\left(L_{0}\right)$, the stalk of $\mathcal{J}\left(h_{\min }\right)_{x_{0}}$ of the multiplier ideal sheaf at $x_{0}$ is the ideal of $\mathcal{O}_{X, x_{0}}$ which is generated by the system of the polynomials

$$
\left\{x_{1}^{p} x_{2}^{q} \mid(p+1, q+1) \in \operatorname{Int}\left(S_{t}\right) \cap \mathbb{Z}^{2}\right\},
$$


where we denote by $\operatorname{Int}\left(S_{t}\right)$ the interior of the set

$$
S_{t}=\left\{\left(\left\langle t m, e_{1}\right\rangle,\left\langle t m, e_{2}\right\rangle\right) \in \mathbb{R}^{2} \mid m \in S\left(L_{0}, h\right)_{\sigma_{1}}\right\} .
$$

For the detail shape of $S_{t}$, see Figure 3 .

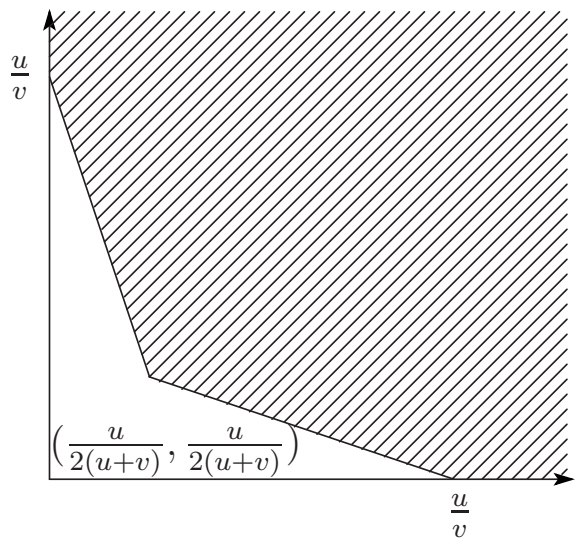

FigurE 3. The shaded area of this figure represents the set $S_{1}$. The set $S_{t}$ is the set of points $p \in \mathbb{R}^{2}$ which satisfies $\frac{p}{t} \in S_{1}$.

The set of the whole jumping numbers $\operatorname{Jump}\left(\psi_{\sigma_{1}} ; x_{0}\right)$ at a point $x_{0} \in \mathbb{P}\left(L_{0}\right)$ can be written as $\operatorname{Jump}\left(\psi_{\sigma_{1}} ; x_{0}\right)=\left\{2 p+(p+q) \frac{v}{u} \mid p, q \in \mathbb{Z}, 1 \leq p \leq q\right\}$, and the singularity exponent $c_{x_{0}}\left(\psi_{\sigma_{1}}\right)$, which is the least number in $\operatorname{Jump}\left(\psi_{\sigma_{1}} ; x_{0}\right)$, satisfies $c_{x_{0}}\left(\psi_{\sigma_{1}}\right)=2\left(1+\frac{v}{u}\right)$.

REMARK 6.3. In Example 6.2, the behavior of the multiplier ideal sheaf $\mathcal{J}\left(\psi_{\sigma_{1}}\right)$ around a point of $\mathbb{P}\left(L_{0}\right)$ coincides with that of the (algebraic) multiplier ideal sheaf $\mathcal{J}\left(\mathfrak{a}^{c}\right)$, where $\mathfrak{a}$ is an ideal generated by $\left(x_{1}^{2(u+v)}, x_{2}^{2(u+v)}, x_{1}^{v} x_{2}^{v}\right)$ and $c$ is the rational number $\frac{u}{2 v(u+v)}$.

This means that the analytic multiplier ideal sheaf $\mathcal{J}\left(\psi_{\sigma_{1}}\right)_{x_{0}}$ has properties same as algebraic multiplier ideal sheaves. For example, it is known that, related to the algebraic multiplier ideal sheaf $\mathcal{J}\left(\mathfrak{a}^{c}\right)$, the set of the whole jumping numbers $\operatorname{Jump}\left(\mathfrak{a} ; x_{0}\right)$ is a discrete subset of the set of rational numbers $\mathbb{Q}$, and has the property so-called "periodicity" in a sufficiently big parts of this set (see [9, 1.12] for details). Indeed, it can be easily checked that $\operatorname{Jump}\left(\psi_{\sigma_{1}} ; x_{0}\right)$ is a discrete subset of $\mathbb{Q}$, and has a "period" $c^{-1}=2 v\left(1+\frac{v}{u}\right)$.

ExAmple 6.4. Second example is the example found out by Nakayama ([14]), which admits no Zariski decomposition even after modifications.

Let us fix an integer $a>1$ and set $L_{0}:=\mathcal{O}_{V}\left(2 F_{1}-4 F_{2}+2 \Delta\right), L_{1}:=\mathcal{O}_{V}((a-$ 1) $\left.F_{1}+(a-1) F_{2}+(a+2) \Delta\right)$, and $L_{2}:=\mathcal{O}_{V}\left((a+3) F_{1}+(a-3) F_{2}+a \Delta\right)$. Then $c_{1}\left(L_{0}\right)=-6\left(l_{1}+\sqrt{3} l_{2}\right), c_{1}\left(L_{1}\right)=6\left(-l_{1}+a l_{3}\right)$, and $c_{1}\left(L_{2}\right)=6\left(-\sqrt{3} l_{2}+a l_{3}\right)$ hold. By these expressions, it turns out that the line bundles $L_{1}$ and $L_{2}$ are ample and, according to Lemma 6.1, that $L$ is also a big line bundle in this case. For this example, see Section 1.

Example 6.5. Finally, we introduce an example which can be proved that admits no Zariski decomposition even after modifications in the almost same way to the case of 
previous Nakayama example, however whose minimal singular metric can be expressed more easily.

Let $L_{0}:=\mathcal{O}_{V}\left(4 F_{1}+4 F_{2}+\Delta\right), L_{1}:=\mathcal{O}_{V}$, and $L_{2}:=\mathcal{O}_{V}\left(-F_{1}+9 F_{2}+\Delta\right)$. Then $c_{1}\left(L_{0}\right)=6\left(l_{1}+3 l_{3}\right), c_{1}\left(L_{1}\right)=0$, and $c_{1}\left(L_{2}\right)=6 l_{1}+10 \sqrt{3} l_{2}+18 l_{3}$ hold. By this expression, it turns out that the line bundle $L_{0}$ is ample and, from Lemma 6.1, that $L$ is also a big line bundle in this case.

The set $\square_{\text {Nef }}\left(L_{0}, h\right)$ in this setting is not rational, but is polyhedral. More precisely, $\square_{\text {Nef }}\left(L_{0}, h\right)$ is the convex closure of the three points $0, e^{1}$, and $\frac{2 \sqrt{6}}{5} e^{2}$ in $M_{\mathbb{R}}$. So, applying theorem 4.7, it immediately turns out that the weight of a minimal singular metric $\psi_{\sigma_{j}}$ satisfies $\psi_{\sigma_{j}} \sim_{\text {sing }} 1$ at any points of $X$ except for the locus $\mathbb{P}\left(L_{2}\right)$, and

$$
\begin{aligned}
\psi_{\sigma_{3}}\left(x_{1}, x_{2}, z\right) & \sim_{\text {sing }} \log \max \left\{\left|x_{0}\right|^{2 \alpha},\left|x_{1}\right|^{2}\right\} \\
& \sim_{\text {sing }} \log \left(\left|x_{0}\right|^{2 \alpha}+\left|x_{1}\right|^{2}\right)
\end{aligned}
$$

at a point $\left(0,0, z_{0}\right) \in \mathbb{P}\left(L_{2}\right)$, where we denote by $\alpha$ the positive irrational number $1-\frac{2 \sqrt{6}}{5}$.

According to the above expression of $\square_{\text {Nef }}\left(L_{0}, h\right)$, the result of Corollary 5.6 can be rewritten as follows. First, it is clear that $\mathcal{J}\left(h_{\text {min }}^{t}\right)$ is trivial at any point in $X \backslash \mathbb{P}\left(L_{2}\right)$. Next, for a point $x_{0} \in \mathbb{P}\left(L_{2}\right)$, the stalk $\mathcal{J}\left(h_{\text {min }}\right)_{x_{0}}$ of the multiplier ideal sheaf at $x_{0}$ is the ideal of $\mathcal{O}_{X, x_{0}}$ which is generated by the polynomials

$$
\left\{x_{1}^{p} x_{2}^{q} \mid(p+1, q+1) \in \operatorname{Int}\left(S_{t}\right) \cap \mathbb{Z}^{2}\right\},
$$

where we denote by $S_{t}$ the set $\left\{\left(\left\langle t m, e_{1}\right\rangle,\left\langle t m, e_{2}\right\rangle\right) \in \mathbb{R}^{2} \mid m \in S\left(L_{0}, h\right)_{\sigma_{3}}\right\}$. For the detail shape of $S_{t}$ in this case, see Figure 4 .

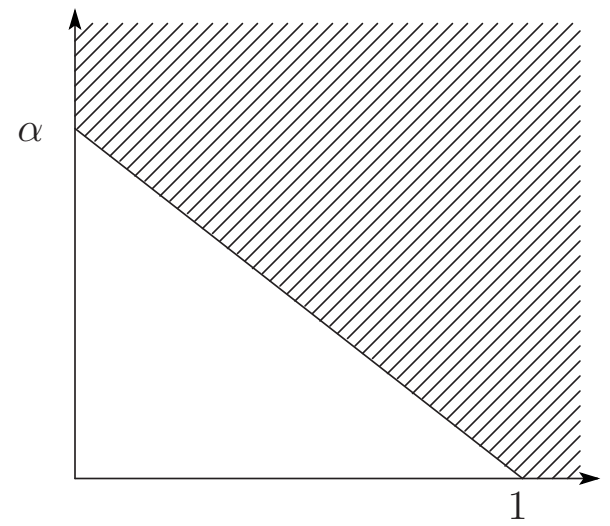

FigurE 4. The shaded area of this figure represents the set $S_{1}$. The set $S_{t}$ is the set of points $p \in \mathbb{R}^{2}$ which satisfies $\frac{p}{t} \in S_{1}$.

Let $x_{0}$ be a point in $\mathbb{P}\left(L_{2}\right)$. In this case, $\operatorname{Jump}\left(\psi_{\sigma_{3}} ; x_{0}\right)$ can be calculated that $\operatorname{Jump}\left(\psi_{\sigma_{3}} ; x_{0}\right)=$ $\mathbb{Z}_{>0} \oplus \frac{1}{\alpha} \cdot \mathbb{Z}_{>0}$, and the singularity exponent can be calculated that $c_{x_{0}}\left(\psi_{\sigma_{1}}\right)=1+\frac{1}{\alpha}$, which is not rational, too. It can easily be proved by using ([14, 2.11]) that $L$ admits no Zariski decomposition even after modifications in this settings. 


\section{REFERENCES}

[1] S. Boucksom, C. Favre and M. Jonsson, Valuations and plurisubharmonic singularities, Publ. Res. Inst. Math. Sci. 44 (2008), no. 2, 449-494.

[2] C. Birkenhake and H. Lange, Second edition, Grundlehren Math. Wiss. 302, Springer-Verlag, Berlin, 2004.

[3] S. Boucksom, Divisorial Zariski decompositions on compact complex manifolds, Ann. Sci. École Norm. Sup. (4) 37 (2004), no. 1, 45-76.

[4] D. A. Cox, J. B. Little And H. K. Schenck, Toric varieties, Grad. Stud. Math. 124, American Mathematical Society, Providence, RI, 2011.

[5] J.-P. Demailly, L. Ein and R. Lazarsfeld, A subadditivity property of multiplier ideals, Michigan Math. J. 48 (2000), 137-156.

[6] J.-P. Demailly, Analytic methods in algebraic geometry, Surv. Mod. Math. 1, International Press, Somerville, MA; Higher Education Press, Beijing, 2012.

[7] J.-P. Demailly and J. Kollär, Semi-continuity of complex singularity exponents and KählerEinstein metrics on Fano orbifolds, Ann. Sci. École Norm. Sup. (4) 34 (2001), no. 4, 525-556.

[8] J.-P. Demailly, T. Peternell and M. Schneider, Pseudo-effective line bundles on compact Kähler manifolds, Internat. J. Math. 12 (2001), no. 6, 689-741.

[9] L. Ein, R. Lazarsfeld, K. E. Smith and D. Varolin, Jumping coefficients of multiplier ideals, Duke Math. J. 123 (2004), no. 3, 469-506.

[10] H. Guenancia, Toric plurisubharmonic functions and analytic adjoint ideal sheaves, Math. Z. 271 (2012), no. 3-4, 1011-1035.

[11] J. Howald, Multiplier Ideals of Sufficiently General Polynomials, ArXiv Mathematics e-prints (mar 2003), arXiv:math/0303203.

[12] R. Lazarsfeld, Positivity in algebraic geometry. I, Ergeb. Math. Grenzgeb. (3) 48, Springer-Verlag, Berlin, 2004.

[13] R. Lazarsfeld, Positivity in algebraic geometry. II, Ergeb. Math. Grenzgeb. (3) 49, SpringerVerlag, Berlin, 2004.

[14] N. Nakayama, Zariski decomposition and abundance, MSJ Mem. 14, Mathematical Society of Japan, Tokyo, 2004.

Mathematical Institute

The University of Tokyo

3-8-1 Komaba, TOKYo

JAPAN

E-mail address: tkoike@ms.u-tokyo.ac.jp 